\title{
The Roles of the Amygdala in the Affective Regulation of Body, Brain, and Behaviour
}

\author{
Marco Mirolli, Francesco Mannella, Gianluca Baldassarre \\ Laboratory of Computational Embodied Neuroscience, \\ Istituto di Scienze e Tecnologie della Cognizione, \\ Consiglio Nazionale delle Ricerche (LARAL-ISTC-CNR) \\ Via San Martino della Battaglia 44, I-00185 Roma, Italy \\ \{marco.mirolli, francesco.mannella, gianluca.baldassare\}@istc.cnr.it
}

\begin{abstract}
Despite the great amount of knowledge produced by the neuroscientific literature affective phenomena, current models tackling noncognitive aspects of behavior are often bio-inspired but rarely bio-constrained. This paper presents a theoretical account of affective systems centered on the amygdala. This account aims to furnish a general framework and specific pathways to implement models that are more closely related to biological evidence. The amygdala, which receives input from brain areas encoding internal states, innately relevant stimuli, and innately neutral stimuli, plays a fundamental role in motivational and emotional processes of organisms. This role is based on the fact that amygdala implements the two associative processes at the core of Pavlovian learning (CS-US and CS-UR associations), and that it has the capacity of modulating these associations on the basis of internal states. These functionalities allow the amygdala to play an important role in the regulation of the three fundamental classes of affective responses (namely, the regulation of body states, the regulation of brain states via neuromodulators, and the triggering of a number of basic behaviours fundamental for adaptation) and in the regulation of three high-level cognitive processes (namely, the affective labeling of memories, the production of goal-directed behaviours, and the performance of planning and complex decision making). Our analysis is conducted within a methodological approach that stresses the importance of understanding the brain within an evolutionary/adaptive framework and with the aim of isolating general principles that can potentially account for the wider possible empirical evidence in a coherent fashion.
\end{abstract}

\section{Introduction}

Since the birth of cognitive sciences in the 1950s, the study of cognitive functions (e.g., perception, attention, memory, planning and decision making) has dominated the sciences of behavior, relegating research on the non-cognitive aspects of behavior (e.g., motivations, moods, emotions) to a marginal role. In general, this is true for all the disciplines dedicated to the study of behavior: for the empirical sciences, from neuroscience to psychology, and for the 'sciences 
of the artificial' (Simon, 1996), from classical artificial intelligence to the new fields of connectionism, autonomous robotics, artificial life, and the simulation of adaptive behavior.

From the point of view of the sciences of the artificial, while classical artificial intelligence research was exclusively dedicated to the study of cognitive capacities, pioneering works on the affective aspects of behavior have been conducted in the fields of artificial life and new robotics from the very beginning (e.g., see Cecconi and Parisi, 1993; Balkenius, 1993; Pfeifer, 1993). The reason for this is related to the significant shift of attention, in the emerging embodied cognition framework, from high level cognitive processes to low level ones, and to the importance attributed to the link between behavior and its biological basis (body, brain, and environment). One of the driving ideas of embodied cognition research is considering behavior and cognition from an adaptive point of view, that is on the basis of the advantages that they can give to organisms in terms of capacity to survive and reproduce. From this perspective, the motivational and emotional aspects of behavior are at least as important as the cognitive ones.

The capacity of survival and reproduction of organisms depends on several different abilities, for example the ability to find food and water, the ability to prevent that the body gets damaged, and to recover when this happens, the ability to find a sexual partner willing to copulate and reproduce, the ability to escape from predators, the ability to find a suitable place for resting and sleeping, and so on. If an agent has to satisfy all these needs, a crucial 'meta-ability' is required, namely the ability to manage the interactions between all these activities. In particular, in each moment the organism must solve the problem of establishing which need it should attend. Affective systems allow organisms to solve precisely this problem, that is to choose which is the activity that has to be accomplished in each moment.

In sharp contrast with what happens in real organisms, artificial systems tend to be designed to accomplish only one or a very few well designed tasks, for example finding the food, or navigating in a complex environment, or categorising a certain object, or grasping and manipulating objects, or coordinating with other agents, and so on. In this kind of agents the problem of selecting which activity to pursue in each moment does not arise because there is only one activity that they can and must pursue in every moment. This is the reason why even in the field of the simulation of adaptive behavior the study of motivations and emotions has always received little attention. In the last years, the realization of the extreme importance that the non-cognitive factors of behavior play in organisms' behavior (Parisi, 2004; Arbib and Fellous, 2004; Canamero, 2005) has significantly boosted the number of researches dedicated to these aspects in the fields of artificial life and autonomous robotics (e.g. Canamero, 1997; Balkenius and Morén, 1999; Murphy, 2002; Mirolli and Parisi, 2003; AvilaGarcia and Canamero, 2004; Montebelli et al., 2008; Venditti et al., 2009).

The relationship between this kind of research and the empirical sciences is typically quite weak, if not completely absent. Generally speaking, the artificial systems developed in these fields are, at most, biologically inspired (bio- 
inspired) but not really biologically constrained (bio-constrained). In other words, the empirical knowledge on the behavior of natural organisms is at most used as a source of interesting ideas, but is not systematically used for constraining the design of artificial systems, nor for testing their empirical predictions. Such a state of affairs has both its motivations and its potential advantages. For example, a certain division of labour between empirical and artificial scientists is necessary. Furthermore, the freedom of not being constrained by available data and knowledge can lead to the development of new ways of framing old problems and of investigating them (i.e. to new 'research paradigms'), and to the discovery of new interesting specific problems and principles. Finally, it must also be considered that a significant proportion of artificial life research has technological rather than scientific aims, and, from a technological point of view, taking into account how natural organisms work is not a need but, at most, an opportunity.

On the other hand, at least from the scientific point of view, the current state of affairs has also important limits. The biological sciences, and the brain sciences in particular, have been producing a huge amount of knowledge on all the aspects that are relevant for understanding organisms' behavior. Furthermore, this empirical knowledge seems to be doomed to grow at an even higher pace in the near future. For this reason, trying to incorporate this knowledge in the design of artificial systems more systematically is likely to produce a fundamental positive impact in our ability to build artificial systems with behavioral capacities more similar to those of natural organisms. This, in its turn, would undoubtedly increase the impact that research on artificial system has on the behavioral and brain sciences. In fact, if it is undeniable that the latter disciplines are producing a large amount of relevant data, it is also true that integrative theories that are able to explain these data and predict new ones are quite scarce. Bio-constrained computational models represent very promising tools for developing these types of theories.

The present work follows an approach which can be called Computational Embodied Neuroscience (or 'CEN', cf. Mannella et al., 2009). According to this approach, behaviour and brain are considered as means through which organisms adapt to the environment in order to increase their chances of survival and reproduction. Consequently, understanding the brain requires understanding how it is structured, functions, and learns in order to produce adaptive behaviour. Moreover, CEN stresses the importance of producing computational models that capture general principles underlying several different behaviours and brain phenomena rather than ad-hoc models addressing only the outcome of single behavioural or neuroscientific experiments. In line with these two principles, our aim here is to furnish both a general framework and several specific suggestions for designing and implementing computational models that have a unifying theoretical scope.

In this paper we contribute to the study of non-cognitive aspects of behavior in artificial systems by providing a theoretical framework on behavior that is based on the available empirical knowledge regarding one of the parts of 
the brain that is at the core of several motivational-emotional systems of higher organisms, namely the amygdala. In particular, we propose a general brain architecture centered on the amygdala, and a number of specific hypotheses on the various functional roles that amygdala plays in the regulation of both affective and cognitive processes. The neuroscientific and behavioral data taken into consideration here mainly refer to the literature on rats. However, the principles proposed and reviewed in the article can probably be extended to more complex mammals (in particular, non-human primates and humans), as most of the brain systems considered here are homologous to those subserving similar functions in primates. The ideas presented here are intended to boost the design and implementation of biologically-constrained computational models like the ones presented by the authors in previous works (e.g., Mannella et al., 2007; Mannella et al., 2008).

The rest of the paper is organised as follows. Section 2 provides a general overview of the amygdala and of the various roles that it plays in the functional organization of adaptive behavior. Section 3 illustrates the three main functioning principles that characterize amygdala as the main locus of classicalconditioning associations. Section 4 presents the three basic functions that amygdala plays in the regulation of affective responses. Section 5 shows the three higher-level functions that amygala plays by interacting with cognitive processes. Finally, section 6 concludes the paper. All the acronyms used throughout the paper are listed in the Appendix (table 6).

\section{Amygdala's roles in adaptive behavior: overview}

The Amygdala (Amg) is an almond-shaped group of nuclei located within each medial temporal lobe of the brain (figure 1). Amg is an important component of several brain subsystems involving the hypothalamus, insular cortex, brain stem, basal forebrain, hippocampus, basal ganglia, and prefrontal cortex, and it has been associated with a wide range of functions including affective regulation, learning, action selection, memory, attention, and perception.

The fundamental hypothesis that underlies the framework proposed in this paper, and schematised in figure 2, is that amygdala is the place where most classical conditioning associations ${ }^{1}$ are acquired on the basis of three basic mechanisms, which roughly correspond to the three major sub-components in which Amg can be divided, that is the central extended amygdala (CEA), the basolateral amygdaloid complex (BLA), and the medial extended amygdala (MEA):

1. CEA associates neutral stimuli (conditioned stimuli, 'CS') directly to basic responses (unconditioned responses, 'UR') that are strictly related to

\footnotetext{
${ }^{1}$ Other classical conditioning associations involving for example basic reflexes like eye blinking are known to be stored in the cerbellum (CB; Thompson et al., 2000). Of course, all classical conditioning processes involve also other parts of the brain beyond Amg and CB, such as the brain stem nuclei and PFC.
} 


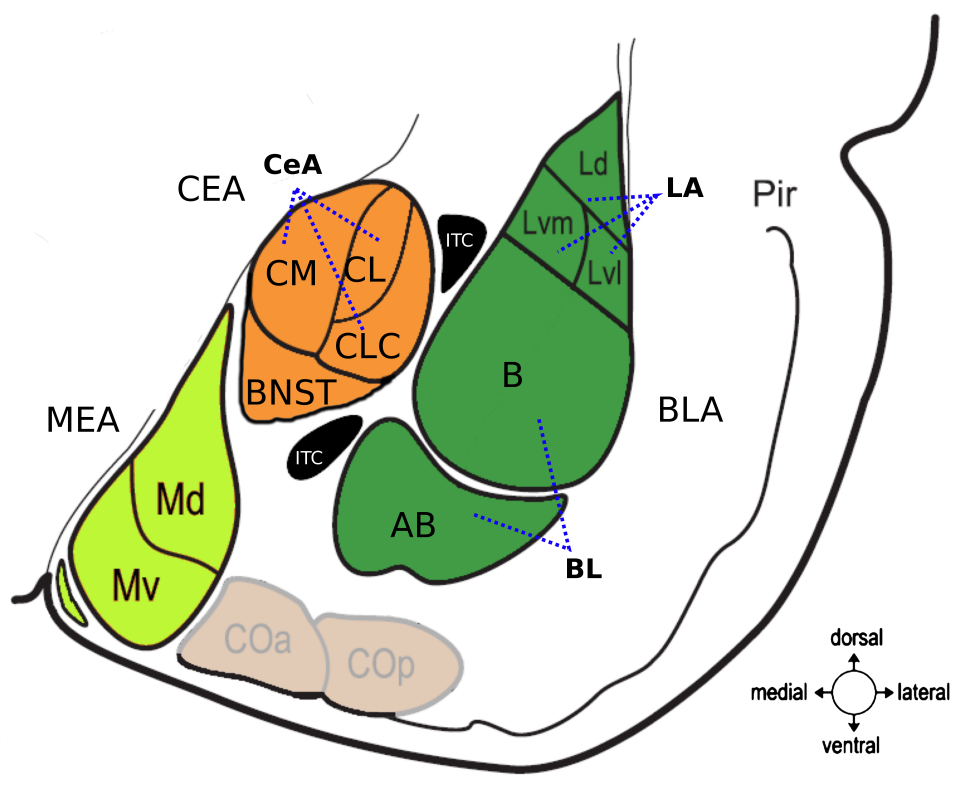

Fig. 1. Nuclei and their subdivisions of rat amygdala. Acronyms: AB (accessory basal amygdaloid nucleus), B (basal amygdaloid nucleus), BL (basolateral amygdaloid nucleus), BLA (basolateral amygdaloid complex), BNST (bed nucleus of the stria terminalis) CEA (central extended amygdala), CM (central medial amygdaloid nucleus), CL (central lateral amygdaloid nucleus), CLC (central amygdaloid nucleus, lateral capsular subdivision), ITC (intercalated nuclei), LA (lateral amygdaloid nucleus), Ld (lateral dorsal amygdaloid nucleus), Lvl (lateral ventrolateral amygdaloid nucleus), Lvm (lateral ventromedial amygdaloid nucleus), MEA (medial extended amygdala), Md (medial amygdaloid nucleus, dorsal part), Mv (medial amygdaloid nucleus, ventral part).

organisms' survival and reproduction on the basis of the experienced cooccurrence of these neutral stimuli and the stimuli that are innately ${ }^{2}$ linked to such basic responses by evolution (unconditioned stimuli, 'US'). The result of this process is the formation of CS-UR associations.

2. BLA associates neutral stimuli (CS) not directly to the basic responses (UR) but rather to the unconditioned stimuli (US) that are innately associated to those responses on the basis of the CS-US co-occurrences experienced during lifetime. The result of this process is the formation of CS-US associations.

\footnotetext{
${ }^{2}$ Note that in the whole paper we will use the expressions 'unlearned', 'unconditioned', or 'innate' to refer to responses that might be either innate or developed during the very first phases of life under strong genetic guidance and general environmental constraints (cf. Arias and Chotro, 2007).
} 
3. MEA modulates CEA's and BLA's representations of stimuli and/or responses (in particular, URs and USs) on the basis of internal body states (i.e. on the basis of the current needs of the organism).

Amg performs these functions on the basis of three main classes of inputs:

1. Body states information, coming from visceral systems, that either constitute unconditioned stimuli or modulate the representations of unconditioned stimuli and responses.

2. Innately relevant information, coming from somatosensory, gustatory, and olfactory systems, that represent unconditioned stimuli.

3. Innately neutral information, coming from visual, auditory, polimodal, and associative areas, that represent stimuli that can be conditioned (i.e. associated to unconditioned stimuli and/or responses).

The basic unconditioned responses (UR) strictly related to survival and reproduction that amygdala is able to associate to innately neutral stimuli constitute, in our view, the fundamental aspects of affective behavior. ${ }^{3}$ These responses can be divided in three basic classes:

1. Regulation of body states, accomplished through the links to the sympathetic, parasympathetic and hormonal systems.

2. Diffuse brain modulation, accomplished through the links to the four main neuromodulatory systems.

3. Triggering of unlearned behaviors, accomplished through the links to the various centers that control such basic behaviors.

Finally, the amygdala has at least three other main outputs, through which it modulates three fundamental cognitive processes on the basis of affective states, thus allowing the emergence of important new cognitive functionalities:

1. Affective labeling, accomplished through the reciprocal connections with the Hip, which is responsible for the encoding and consolidation of episodic memories: these connections allow Amg to include motivational and emotional elements in such memories and to enhance their encoding and recall.

2. Goal-directed behavior, accomplished through the connections targeting the NAccC-PL loop, which is responsible for the higher-level stages of actionselection: these connections allow the affective state of an organism to influence the selection of behaviors acquired through operant conditioning.

3. Planning and decision-making, accomplished through the reciprocal connections with PFC, which hosts many important cognitive processes such as working memory, attention, and prediction: these connections allow affective states to influence the processes taking place in PFC, thus guiding topdown attention, monitoring of action execution, complex decision making, and planning.

\footnotetext{
${ }^{3}$ Throughout the paper we will use the word 'affective' to refer to both motivational and emotional aspects of behaviour.
} 


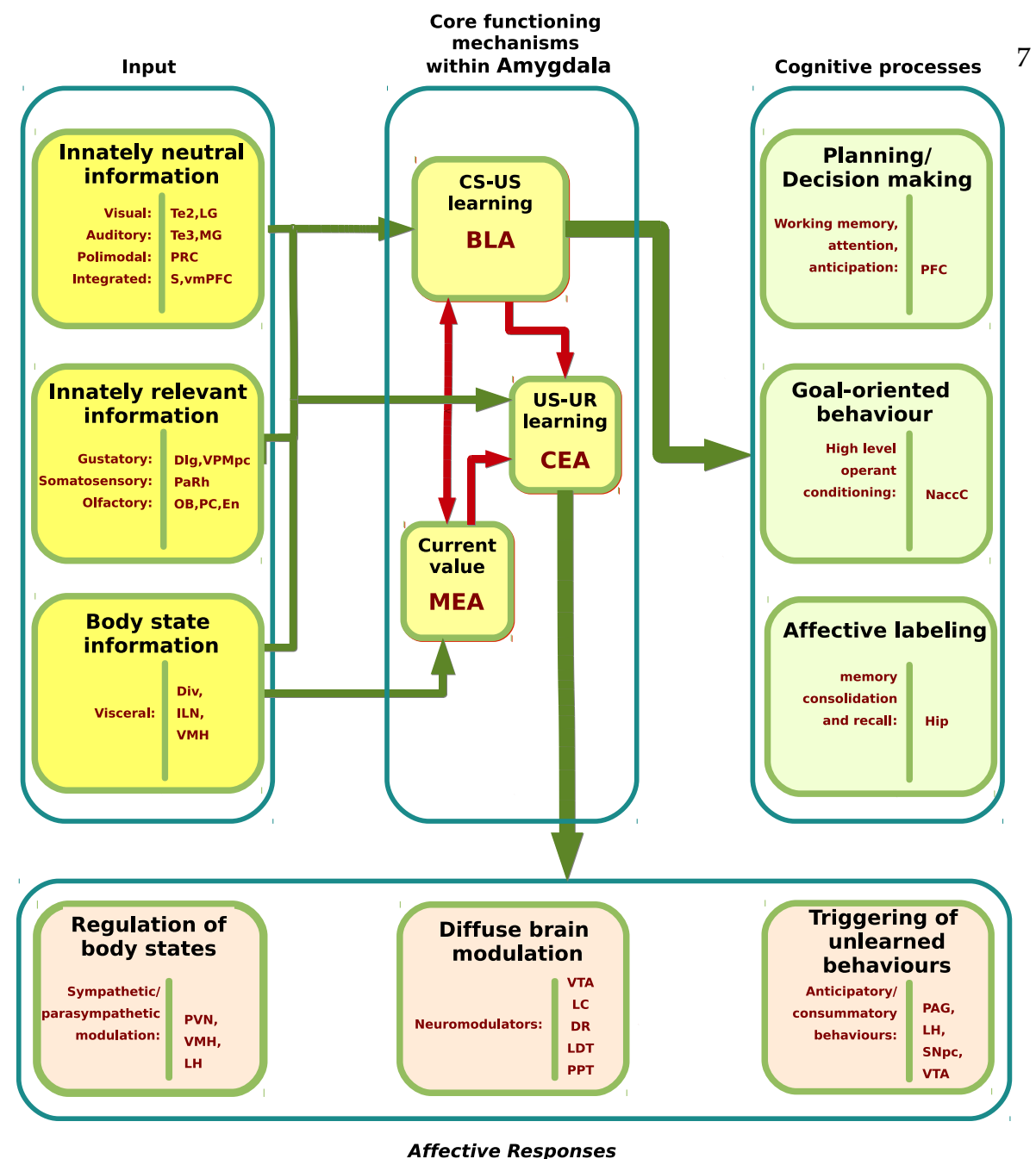

Fig. 2. Scheme indicating the main functions played by Amg and the main brain anatomical areas which implement such functions. The scheme indicates the three main classes of input received by Amg, the three basic mechanisms it implements, the three types of output through which it regulates the affective responses, and the three main influences it exerts on higher cognitive processes (see text for details). Ancronyms: BLA (basolateral amygdaloid complex), CEA (central extended amygdala) DIg (disgranular insular cortex, gustatory part), DIv (disgranular insular cortex, visceral part), DR (dorsal raphe), En (endopiriform nucleus), Hip (hippocpampus), ILN (infralaminar nucleus), ITC (intercalated nuclei), LC (locus coeruleus), LDT (laterodorsal tegmental nucleus), LG (lateral geniculate nucleus), LH (lateral hypothalamus), MEA (medial extended amygdala), MG (medial geniculate nucleus), NaccC (nucleus accumbens core), OB (olfactory bulb), PAG (periaqueductal gray), PaRh Parietal rhinal cortex, PC (piriform cortex), PFC (prefrontal cortex), PPT (pedunculopontine tegmental nucleus), PRC (perirhinal cortex), PVN (paraventricular nucleus of hypothalamus), S (subiculum), SNpc (substantia nigra, pars compacta), Te2 (temporal cortex, area 2), Te3 (temporal cortex, area 3), VMH (ventromedial hypothalamus), VPMpc (ventral posteromedial nucleus, parvicellular part), VTA (ventral tegmental area), vmPFC (ventromedial prefrontal cortex). 


\section{The roles of the amygdala in classical conditioning}

Individual learning plays a fundamental role in the adaptive behavior of organisms, especially in the most sophisticated ones like mammals. For this reason, animal psychology has devoted great efforts to the study of learning processes. In particular, in the last century a huge body of empirical data has been collected around the two main experimental paradigms of 'classical conditioning' and 'instrumental conditioning'.

Classical conditioning (or Pavlovian conditioning) refers to an experimental paradigm in which a certain basic behaviour such as salivation or approaching (UR), which is innately linked to a biologically salient stimulus such as food ingestion (US), gets associated to a neutral stimulus like the sound of a bell (CS), after the neutral stimulus is repeatedly presented before the appearance of the salient stimulus. Such acquired associations, as mentioned in section 2, are briefly referred to as 'CS-US' or 'CS-UR' associations (Pavlov, 1927; Lieberman, 1993, see below).

Instrumental conditioning (or operant conditioning) refers to an experimental paradigm in which an animal, given a certain stimulus, such as a lever in a cage (the stimulus, ' $\mathrm{S}$ '), learns to produce a particular action such as pressing the lever (the response, ' $R$ '), if the performed action consistently leads to a rewarding outcome, such as the access to food. In this case, the acquired associations are briefly referred to as 'S-R' associations (Thorndike, 1911; Skinner, 1938; Domjan, 2006).

The current most influential models of conditioning phenomena, those based on temporal-difference reward prediction error (Schultz et al., 1997; Sutton and Barto, 1998; Schultz and Dickinson, 2000; Schultz, 2002), suffer from various limitations (cf. Redgrave et al., 1999; Dayan, 2002; Redgrave and Gurney, 2006; Berridge, 2007; Mannella et al., 2007). For example, they tend to conflate classical and instrumental conditioning, and they do not take into account the influences of internal states on the acquisition and expression of conditioned responses. One of the reasons of these limits is that such models have been developed within machine learning with the aim of building artificial systems that can autonomously learn to perform actions that are useful for the user. As a result, they are more suitable for investigating instrumental conditioning phenomena but less adequate to explain Pavlovian ones (Dayan and Balleine, 2002; O'Reilly et al., 2007).

From the scientific point of view, the available empirical knowledge indicates that basal ganglia represent the main neural substrate where the S-R associations acquired through instrumental conditioning are stored (Barto, 1995; Bar-Gad et al., 2003; Yin and Knowlton, 2006), whereas amygdala represents the main neural substrate where the associations acquired through Pavlovian conditioning are stored (Baxter and Murray, 2002; Cardinal et al., 2002).

A crucial question on classical conditioning regards the nature of the acquired association between the CS and the UR: is this association direct (CSUR), as Hull (Hull, 1943) suggested, or does it pass through the unconditioned stimuli (CS-US-UR), as Pavlov himself seemed to claim (Pavlov, 1927)? The 
long-lasting debate on this topic (Lieberman, 1993) seems now to have settled in favor of both hypotheses: there is in fact strong empirical evidence supporting the co-existence of both CS-UR and CS-US associations (Dayan and Balleine, 2002). In particular, the available empirical evidence suggests that CEA stores CS-UR associations, whereas BLA stores CS-US associations (Cardinal et al., 2002; Mannella et al., 2008). The rest of this section describes our hypotheses on the specific mechanisms that Amg might exploit to implement these two basic functionalities and to modulate them on the basis of the current internal states.

\subsection{CEA as the locus of US-UR associations}

All animals are genetically endowed with a set of basic responses that have a high direct relevance for their survival and reproduction. These responses belong to three classes: (a) internal responses directed to regulate the states of the body of the organism (discussed in section 4.1); (b) neuromodulatory responses that influence the general states of the brain or the relative activity of different parts of it (discussed in section 4.2); (c) basic behavioral responses (discussed in section 4.3). These responses are innately linked to specific stimuli so that when a given stimulus is perceived, the appropriate responses are automatically triggered. For example, when an animal perceives the odour of a predator its heart-rate speeds up (body), its general alertness increases (brain), and its body might freeze (behavior).

In the case of complex animals living in a complex and dynamic world, it is not possible for evolution to a-priori associate the appropriate responses to all the possible stimuli that the animals can encounter during life. The solution that evolution found to this problem is endowing animals with a learning system that associates the basic unconditioned responses to the conditioned stimuli that are systematically experienced in conjunction with (or as predecessors of) the relative basic unconditioned stimuli. CEA is the part of the brain that learns and stores most of these CS-UR associations. In fact, CEA has been shown to be necessary for the acquisition and expression of both aversive (e.g., freezing) and appetitive (e.g., approaching) conditioned reactions (Shi and Davis, 1999; Nader et al., 2001; Lanuza et al., 2004; Hatfield et al., 1996; Parkinson et al., 2000). For example, Hatfield et al. (1996) showed that CEA lesions impair the capacity of rats to acquire the association between an unconditioned response (orienting) and a conditioned stimulus (light), while lesions of BLA do not affect this capacity.

CEA is able to make these associations thanks to its pattern of connectivity (see figure 3). From the efferent side, CEA constitutes the main output gateway of Amg, sending projections to several brain areas that control all three kinds of unlearned responses (affecting the body, the brain, and basic behaviors, see section 4). On the afferent side, CEA receives external projections from both the brain areas having information about unconditioned stimuli (i.e. visceral, somatosensory, olfactory and gustatory) and from those having information about conditioned stimuli (i.e. visual, auditory, polimodal, and associative) (McDonald, 1998; Jolkkonen and Pitkänen, 1998; Sah et al., 2003). Furthermore, 
both these kinds of information arrive to CEA also indirectly, via its afferent projections from BLA, in particular from LA, which constitutes the principal input gateway of the whole Amg: in fact, also LA receives information on both innately relevant and on neutral stimuli required for classical conditioning associations (McDonald, 1998; Pitkänen et al., 2000; Sah et al., 2003; Maren, 2005; Paré et al., 2004). CS-UR associations involve both the internal (from BLA) and the external (from the rest of the brain) afferent projections to CEA since LA lesions sometimes impede these associations (Lanuza et al., 2004; Blair et al., 2005), while in other cases they do not (Hatfield et al., 1996).

Figure 3 provides a schematization of CS-UR learning in CEA. CS-UR associations can be acquired through the modification of the afferent connections going from conditioned stimuli (CSs), represented either within LA or outside Amg, to an unlearned responses (URs) that are triggered by the unconditioned stimuli (USs) which are systematically paired with the given CSs (the scheme is both a simplification and an elaboration of the computational model that the authors used for simulating experiments on second-order conditioning in normal and BLA lesioned rats, see Mannella et al., 2008; see also section 4.2).

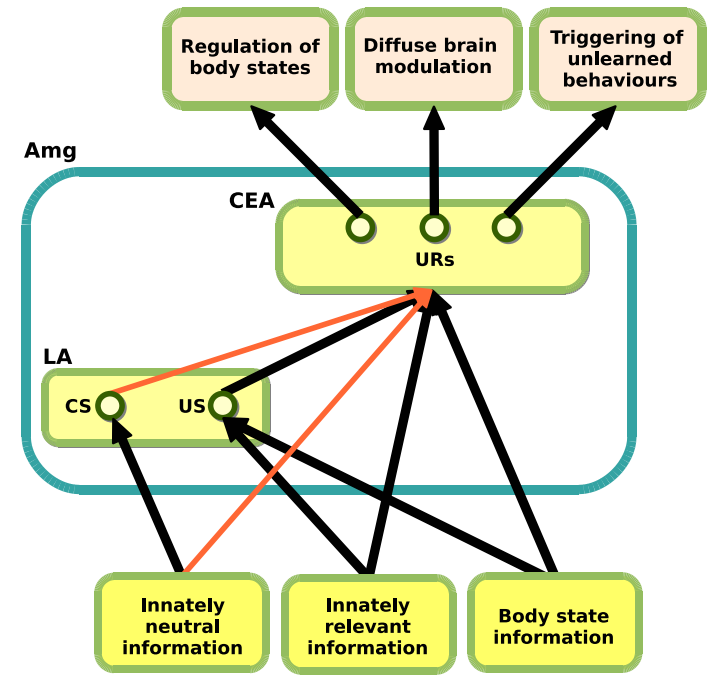

Fig. 3. CEA: schematization of the learning of CS-UR associations (thin arrows) on the basis of the pre-existing unlearned US-UR associations (thick arrows). Ancronyms: Amg (amygdala), CEA (central extended amygdala) LA (lateral amygdaloid nucleus).

\subsection{BLA as the locus of CS-US associations}

Direct CS-UR associations have a clear adaptive advantage, but have also two limitations. First, among the unconditioned responses that can be triggered by 
CEA (and hence can be associated to conditioned stimuli through CS-UR learning) there is not the production of learning signals, for example the production of the phasic dopaminergic bursts (Schultz et al., 1997; Schultz and Dickinson, 2000) or noradrenergic bursts (Berridge and Waterhouse, 2003) that are thought to be at the basis of learning. In fact, CEA has mainly inhibitory GABAergic efferent connections (McDonald, 1998), for example reaching VTA producing dopamine (DA) or LC producing norepinephrine (NE). When these connections reach target areas they tend to produce modulatory tonic signals instead of the phasic signals that are thought to trigger learning (cf. section 4.2). Moreover, while CEA lesions disrupts the capacity to show CS-UR associations, they do not disrupt the capacity of conditioned stimuli to be used as reinforcements in second-order conditioning (Hatfield et al., 1996). The second limitation of having only CS-UR associations is that the direct association of stimuli with basic reactions would not allow the use of conditioned stimuli for influencing more complex cognitive processes. In order to overcome these limitations the brain evolved a mechanism to link neutral stimuli to unconditioned stimuli, so that the presentation of a CS can recall the associated US and both trigger the phasic bursts of neuromodulators driving learning and modulate high-level cognitive processes.

There is plenty of evidence that BLA is the part of the brain that learns and stores CS-US associations. In fact, BLA has been shown to be necessary for both the modulation of high-level cognitive processes by motivational/emotional states (see section 5) and the manifestation of second-order conditioning phenomena, in which a conditioned stimulus (e.g., a light) is used as a secondary reward in extinction (i.e. without first order reward) in order to condition a second neutral stimulus (e.g., a tone) (Hatfield et al., 1996). The acquisition of rewarding effects by conditioned stimuli that have been systematically experienced with unconditioned stimuli is likely to depend on BLA excitatory glutamatergic connections (which are suitable for causing phasic responses) that directly or indirectly target mid-brain neurons that produce neuromodulators (see, for example, figure 4 for DA, which is produced by neurons in VTA and $\mathrm{SNpc}$ ).

BLA is able to make these associations thanks to its connectivity (figure 4). As discussed above, LA (which is part of BLA) is the main input gateway of the whole Amg, receiving information both regarding USs (from visceral, gustatory, olfactory, and somatosensory areas) and regarding CSs (from visual, auditory, polimodal and associative areas). Furthermore, the areas of BLA that receive these two kinds of information are reciprocally interconnected, thus permitting the associations between CSs and USs to take place.

Interestingly, the internal connectivity within BLA suggests that the convergence between CSs and USs takes place in two sites organised in sequence: (a) at the level of Lv (which is a part of LA) visceral, somatosensory and gustatory information (USs) converge with auditory and visual information (Romanski et al., 1993; Pitkänen et al., 1995; Maren, 2005); (b) at the level of BL, information about USs converges with highly integrated polimodal information from hip- 
pocampal, cortical associative and cortical prefrontal areas (McDonald, 1998; Pitkänen et al., 2000; Price, 2003; Sah et al., 2003). This hierarchy in BLA's internal connectivity suggests that USs can be associated with stimuli of different levels of complexity: from the simplest unimodal stimuli that are typically used in classical conditioning experiments (e.g., lights or tones), to complex objects, context, or places, like in conditioned place preference experiments (Hiroi and White, 1991; McDonald and White, 1993).

Finally, the representations of USs, which can be recalled by associated CSs, can control three different classes of systems thanks to different sets of BLA efferent projections (see figure 4). First, projections to Hip (McDonald, 1998; Richter-Levin, 2004), NAcc (Cador et al., 1989; Pitkänen et al., 2000), and PFC (Rolls, 2000; Sah et al., 2003) allow conditioned stimuli to influence cognitive functions (see section 5 below). Second, projections to neuromodulatory systems (e.g., VTA and SNpc for DA, reached by BLA through LH and PPT (McDonald, 1998; Pitkänen et al., 2000) allow conditioned stimuli to act as secondorder reinforcements by producing the activity bursts that are supposed to drive learning. Third, intra-amygdaloid projections to CEA (Sah et al., 2003) allow CSs to trigger all the URs normally triggered by the associated USs.

Figure 4 represents a schematization of the BLA functioning: CS-US associations are learned through the modification of the BLA lateral connections which link the representations of innately neutral stimuli with those of unconditioned stimuli which innately trigger unconditioned responses. This scheme is both a simplification and an elaboration of the computational models that we used for simulating real experiments on both second-order conditioning (Mannella et al., 2008) and devaluation (Mannella et al., 2007, 2009; see also section 5.2).

Finally, it is important to mention that all the CS-US associative properties which we have so far ascribed to BLA likely depend on a wider system formed by BLA and OFC, a region of PFC with which BLA exchanges dense reciprocal interconnections. In fact, experiments involving lesioning either BLA or OFC show that it is very difficult to dissociate the functions of BLA and those of OFC (Schoenbaum et al., 2007; Pickens et al., 2005; Roesch and Schoenbaum, 2006), although recent investigations are starting to show that OFC is more closely involved with working memory processes whereas BLA is more closely related to learning CS-US associations (Schoenbaum et al., 2003, cf. also section 5.3).

\subsection{MEA as the locus of the modulation of USs and URs by internal states}

The mechanisms by which an organism can learn to associate innately neutral stimuli to innately specific responses strictly linked to survival and reproduction are really useful only if there is a way to modulate these associations according to the current internal state of the organism. For example, let us consider feeding behavior. Even in presence of the stimuli that have been repeatedly experienced as predictive of food, it is useful to trigger all the responses related to feeding (e.g., orienting, approaching, salivating, etc.) only when the energy level of the organism is low (i.e. when the organism is hungry), but not when the organism is satiated. If this does not happen, when encountering a 


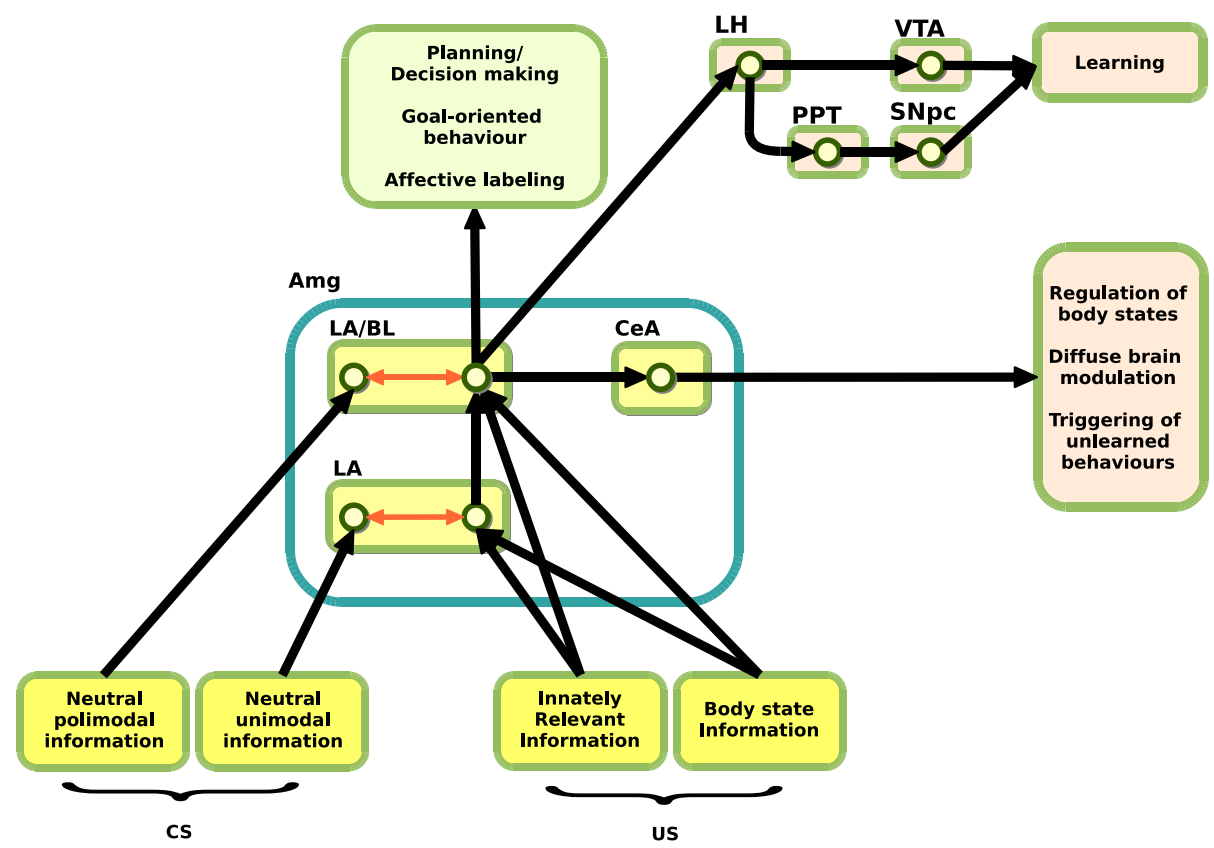

Fig. 4. BLA: schematization of the learning of CS-US associations (thin arrows) on the basis of the pre-existing unlearned US-UR associations (thick arrows). Ancronyms: Amg (amygdala), BL (basolateral amygdaloid nucleus), CeA (central amygdaloid nucleus), LH (lateral hypothalamus), PPT (pedunculopontine tegmental nucleus), SNpc (substantia nigra, pars compacta), VTA (ventral tegmental area).

place where there is plenty of food an organism would indefinetely continue to produce feeding related responses, thus risking, for example, to die of thirst. As discussed in section 1, regulating which kind of activity an organism pursues in each moment is exactly the function of a well designed motivational system. The regulation of organisms' activities on the basis of its current internal state is what makes organisms' behavior proactive (i.e. controlled by their needs) rather than reactive (i.e. completely determined by external stimuli).

The need to flexibly and efficiently modulate basic unconditioned responses on the basis of the current state of the body might even constitute one of the most important reasons why the CS-US system in BLA has evolved to supplement the probably more basic CS-UR system in CEA. In order to understand why, let us consider the case of food devaluation. There can be two types of devaluation: 'temporary', for example when the organism is satiated, and 'permanent', for example when a food turned out to be toxic (e.g., its ingestion was followed by nausea).

In principle, temporary devaluation might be efficiently faced even with only a CS-UR system: if the current state of the body directly modulates the 
unconditioned responses related to feeding (e.g., orienting, approaching, salivating), then these responses could be temporarily blocked regardless of the stimulus that would trigger them (be it unconditioned or conditioned). But the same solution is not viable for permanent devaluation: an animal cannot permanently block all feeding responses, otherwise it would die of starvation. Moreover, for an animal which possesses only a CS-UR system, also a solution in which permanent devaluation is done at the level of the US is satisfying. In fact, such a solution could not prevent the triggering of preparatory feeding responses by those CSs that have been associated with the devaluated food, with the result of an inefficient activity directed towards the dangerous food.

A CS-US system allows solving this problem. The reason is that in such a system devaluation can be done at the level of the US. In this way, the devaluated US can inhibit the URs that are innately associated to it without preventing other stimuli to trigger those responses when neither the devaluated US nor the CSs linked to it are present. This solution can work equally well for both temporary and permanent devaluation.

While a considerable amount of empirical research has been dedicated to understanding the roles of CEA and BLA in CS-(US)-UR associations, much less work has been done to clarify the exact neural mechanisms through which unconditioned responses are modulated by the internal states of organisms. The available empirical evidence suggests that this is exactly the function of the third main group of Amg nuclei, namely MEA. First of all, there is evidence that MEA does indeed play a role in regulating the triggering of basic behaviors on the basis of the state of the body: for example, lesions to MEA have been shown to produce disturbances to feeding behavior that lead to obesity (King, 2006), which depends on the incapacity of regulating the triggering of an unconditioned behavior (e.g., feeding) on the basis of the current state of the body (e.g., the level of hunger). Second, MEA has just the right kind of connectivity for supporting this modulatory function (see figure 5). In fact, MEA is reciprocally connected to Hyp (in particular VMH, LH, and PVN: Pitkänen et al., 2000; De Olmos et al., 2004), which is the main center which processes the information regarding the current states of the body. Moreover, MEA sends efferent inhibitory GABAergic projections to both CEA and BLA (Pitkänen et al., 1997; De Olmos et al., 2004), and receives excitatory connection from BLA.

Figure 5 represents a schematization of how MEA could modulate both US and UR representations in BLA and CEA on the basis of the current body states. Once a representation of US in BLA gets activated (either directly, or via the activation of an associated CS), it tends to activate the respective representation in MEA. If the parts of the brain representing the state of the body (e.g., the Hyp) inform MEA that that US is devaluated, the corresponding unit in MEA gets fully activated and can inhibit both the representation of the stimulus in BLA and the representations of the corresponding URs in CEA.

Within this scheme, temporary devaluation (e.g., caused by free feeding and satiation) might be implemented by inhibitory connections from MEA to CEA and BLA which have both fast learning and fast forgetting. In this way, for 
example, when the organism is satiated these connections might strengthen and inhibit the related US and UR, whereas when the organism is hungry they might decrease thus allowing the triggering of feeding-related responses. Permanent devaluation might be implemented by other similar connections which have a fast learning but a slow forgetting.

This schema might also explain a last important phenomenon, known as incentive learning (Balleine and Dickinson, 1998; Balleine and Killcross, 2006), shown in experiments where the current value of a US (say 'USa') is transferred to another US (say 'USb') only if the animal can experience USb after the devaluation of USa. The explanation is that if USb is not re-experienced after devaluation of USa the connections from its representation in MEA and the one in BLA (and the relative URs in CEA) has not grown up, thus not inhibiting the responses to the associated CSs. On the other hand, as soon as USb is re-experienced when the animal is in a satiated condition, the inhibitory connections would immediately grow, thus preventing the associated CS to trigger the unconditioned responses.

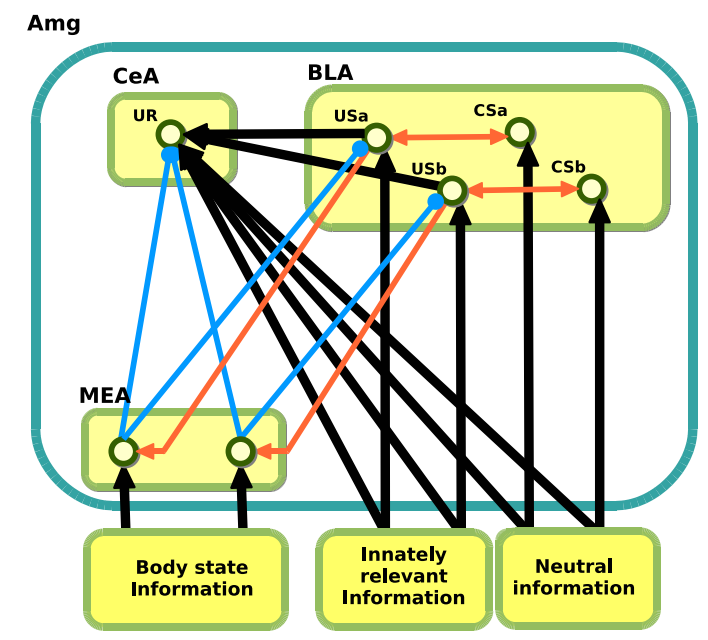

Fig. 5. MEA: schematization of the modulation of Pavlovian associations based on internal states, plastic connections (thin arrows) and innate connections (thick arrows). Circle edges denote inhibitory connections whereas arrow edges denote excitatory connections. Ancronyms: Amg (amygdala), BLA (basolateral amygdaloid complex), CeA (central amygdaloid nucleus), MeA (medial amygdaloid nucleus).

\section{The roles of the amygdala in affective processes}

According to the framework presented in section 2 Amg has evolved to efficiently associate several innate responses (URs) that are directly important 
for organisms' survival and reproduction to innately neutral stimuli (CSs) that are repeatedly experienced as predictors of the stimuli (USs) that trigger those responses. This section illustrates in detail the operation of this fundamental function of Amg with respect to the three classes of unconditioned responses: regulation of body states (section 4.1), diffuse brain modulation (section 4.2), and triggering of unlearned behaviors (section 4.3). Recall from section 2 that the processes regulating these three kinds of basic responses are here assumed to be essential components of motivations and emotions.

\subsection{Regulation of body states}

The regulation of body states based on external events is a fundamental functionality for complex organisms that have several needs to satisfy. For example, if an organism is going to eat, it is useful for it to prepare for digestion by salivating and increasing the blood flow to the gut. But if a predator suddenly appears, the same organism has to prepare its body for fighting or fleeing, for example by suddenly redirecting the blood flow to the muscles, by increasing the heart rate, by increasing glucose release, etc.

Thanks to its associative properties, Amg can trigger these body regulations not only in response to innately relevant stimuli but also in response to stimuli which are constantly experienced as preceding or accompanying them. The adaptive advantages of these capability are evident: body states can be triggered in advance with respect to the events that make them useful. These processes are captured in the laboratory by the classical experiments of Pavlov, in which a dog learns to prepare its body for digestion by salivating in advance when it hears a bell that has been systematically associated with the delivery of food.

Many of these body regulations take place via the influence of the 'autonomic nervous system' (ANS), which includes the sympathetic and parasympathetic nervous systems (SNS and PSNS, respectively). The SNS is always active at a basal level ('sympathetic tone') and becomes more active during times of stress. Under stressing conditions the SNS prepares the body for fight-or-flight responses by enhancing arousal and energy generation and inhibits digestion. In particular, it diverts blood flow away from the gastro-intestinal tract and skin via vasoconstriction, enhances blood flow to skeletal muscles and lungs, dilates bronchioles of lungs, increases heart rate, dilates pupils, inhibits peristalsis (Davis and Whalen, 2001; Iversen et al., 2000). The PSNS has a complementary function with respect to the SNS: in general, it can be said to prepare the body for a rest-and-digest mode of behaviour in that it promotes calm action and digestion. In particular, in absence of salient stimuli and compelling needs, PSNS dilates blood vessels leading to the gastro-intestinal tract, constricts the bronchiolar diameter in lungs, diminishes heart rate, causes constriction of pupils, stimulates salivary gland secretion, accelerates peristalsis, and causes erection of genitals (Iversen et al., 2000).

Amg influences the SNS and the PSNS mainly via CEA (Davis and Whalen, 2001). In particular CEA influences the SNS via efferent connections directed to 
various nuclei of Hyp, mainly LH, PO, and PVN (Jolkkonen and Pitkänen 1998; Knapska et al. 2007; see figure 6) which in turn send efferent connections to the brain-stem and the spinal-cord (Davis and Whalen, 2001). Through its connections to LH, CEA can influence thirst and hunger (that is, the perception of the internal lack of water and food). Through its connections to PO, it can modulate urination, heart rate, and blood pressure. Through its connections to PVN, it can influence gastric reflexes, blood pressure, and temperature regulation.

CEA influences the PSNS via connections to DMX, AMB, and MEV (Knapska et al. 2007; figure 6). These allow CEA to contribute to regulate body functions such as salivation, lacrimation, digestion, urination, and defecation.

The innervations to PVN are also very important as they allow CEA to control the hypothalamic-pituitary-adrenal axis, which, via the Pituitary gland (or 'hypophysis'), has a major role in the regulation of the network of body hormones (Iversen et al., 2000). Through this axis CEA can influence virtually all internal processes, including water retention, blood pressure, temperature regulation, male aggression, uterine contractions and lactation, the production of extrogens, analgesy, and metabolism of nutrients (Iversen et al., 2000).

\subsection{Diffuse brain modulation}

Like the regulation of the body, the regulation of diffuse brain states plays a central role for organisms that have to satisfy several different needs. In fact, the performance of different activities requires the differential involvement of different brain areas and the functioning of such areas with different modes. The modulation of brain activity is accomplished in two ways: (a) indirectly, via the body, through the activation of endocrine glands that release hormones in the blood (hormones regulate both the body and brain states); (b) directly, via the activation of ancient nuclei of neurons that release the four principal neuromodulators: the monoamine serotonin (5-HT), and the three catecholamine dopamine (DA), norepinephrine (NE; also named 'noradrenaline'), and acetylcholine (ACh). The neuromodulators are produced in two main ways, that tend to have different effects on target neurons:

1. Tonic production involves a prolonged populational activation of the neuromodulatory neurons, typically induced via their diffused GABAergic disinhibition, which leads to the accumulation of the neuromodulator in the extrasynaptic space. The main effect of tonic production of neuromodulators is the general modulation of the targeted areas.

2. Phasic production involves a high but short activation of the neuromodulatory neurons, typically induced via their glutammaergic direct activation, which leads to fast but temporary high increase of neuromodulator in the intra-synaptic space. Phasic production of neuromodulators is supposed to have an important role in learning processes (see the case of DA, below) and for quick regulation of brain states when speed is paramount (e.g., to face a predator). 


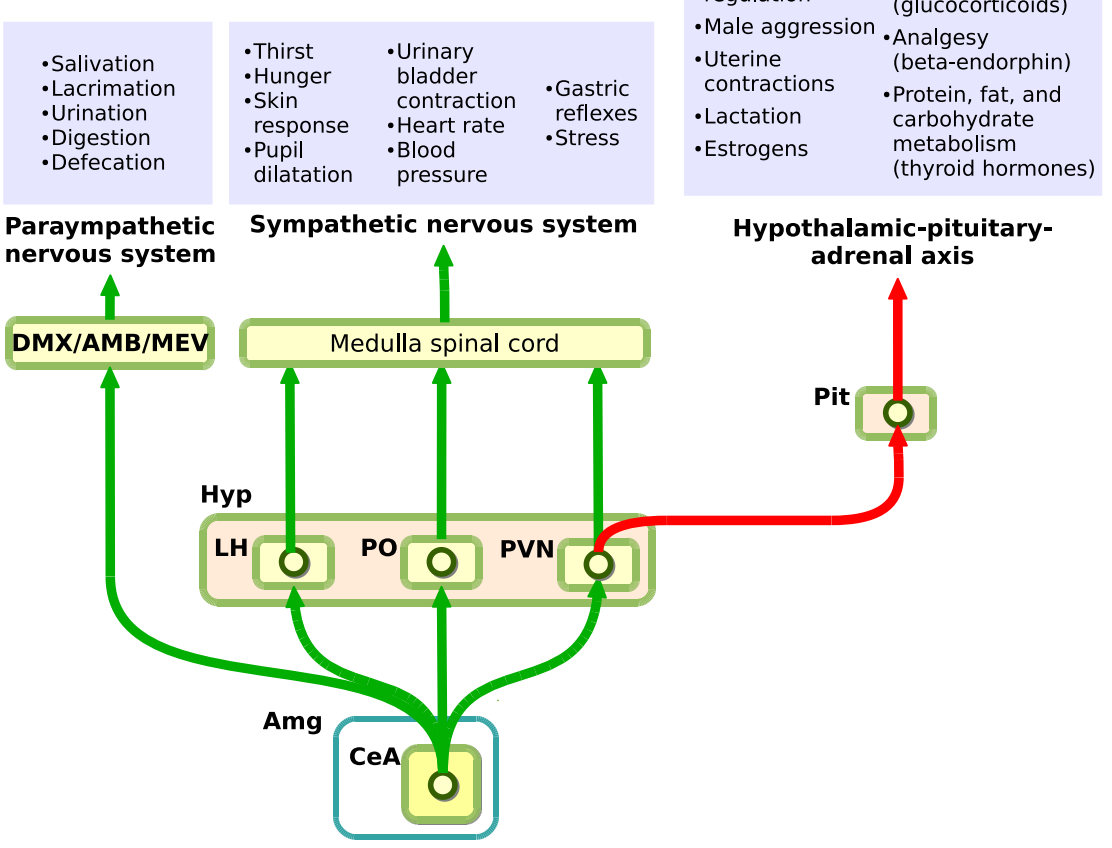

Fig. 6. Body states regulation: schematization of how amygdala contributes to regulate the body states via the sympathetic, parasympathetic and hormonal systems. Ancronyms: AMB (nucleus ambiguus), CEA (central extended amygdala), DMX (dorsal motor nucleus of the vagus nerve), Hyp (hypothalamus), LH (lateral hypothalamus), MEV (midbrain trigeminal nucleus), PO (preoptic nucleus of hypothalamus), PVN (paraventricular nucleus of hypothalamus), Pit (pituitary gland).

Even with respect to the brain modulation, the core function of Amg is based on its capacity to transfer the effects genetically associated to biologically salient stimuli (US) to innately neutral stimuli (CS). So, for example, the increased levels of stress- and alertness-related regulations innately associated to the perception of a predator can be transferred to the type of noises which preceded the attack, or to the sight of the place where the attack took place.

The Amg exerts brain modulations mainly via CEA (Davis and Whalen, 2001), which is connected to the main brain nuclei that are responsible of the production of the neuromodulators. One important exception is the modulation by BLA of the burst firing of the dopamine neurons via glutamatergic projections to LH (Petrovich et al. 2002, 2005; see also section 3.2). 


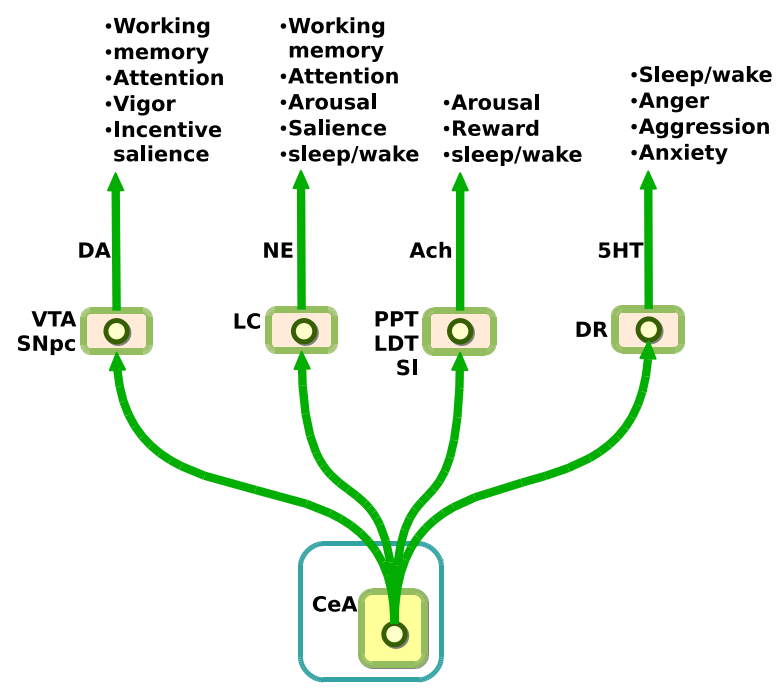

Fig. 7. Brain states regulation: schematization of how amygdala contributes to regulate brain states via the diffused action of neuromodulators. Ancronyms: VTA (ventral tegmental area), SNpc (substantia nigra pars compacta), LC (locus coeruleus), PPT (pedunculopontine tegmental nucleus), LDT (laterodorsal tegmental nucleus), SI (substantia innominata), DR (dorsal raphe), ACh (acetylcholine), DA (dopamine), NE (norepinephrine), 5HT (serotonine).

Amg modulates the production of DA by influencing the two main centers of dopaminergic neurons: VTA, which reaches NAcc and PFC (Fudge and Haber, 2000; Fudge and Emiliano, 2003), and SNpc, which sends projections principally to BG, especially its DLS and DMS components (Han et al., 1997; Lee et al., 2005). Tonic DA enhances the general level of processing of PFC, thus enhancing working memory and attention (Phillips et al., 2008). Moreover, tonic DA in NAcc seems to modulate organisms' vigor, that is the number of actions that the animal performs in a given amount of time and the involvement of energy spent in their execution (Niv et al., 2006; Floresco, 2007). Phasic DA signals the positive/negative salience of stimuli that is at the basis of learning processes taking place within BG (Schultz, 2002; Surmeier et al., 2007) and vmPFC (Otani et al., 2003).

Amg modulates the production of NE through LC, which innervates virtually the whole cortex, BG, Th, Hyp, Hip, CB, and the spinal cord (Berridge and Waterhouse, 2003; Aston Jones and Cohen, 2005). NE plays an important function in the regulation of the sleep/wake cycle, attention, arousal, and work- 
ing memory, on the basis of the novelty of perceivevd stimuli and contexts (Berridge and Waterhouse, 2003). ${ }^{4}$

Amg regulates the production of Ach mainly via PPT, LDT (Semba and Fibiger, 1992; Knapska et al., 2007), and SI (Jolkkonen et al., 2002), which innervate the brainstem, Amg, Hip, and PFC. In the central nervous system, Ach is known to modulate the sleep/wake cycle, synaptic plasticity (LTP), general excitability, arousal, and reward (Chen et al., 2006). ${ }^{5}$

Both directly and via LH and PAG (Peyron et al., 1998; Bandler et al., 2000), Amg regulates the production of 5-HT by the DR, which innervates BG (including NAcc), Th, Hyp, Hip, Amg, and virtually the whole cortex (Barnes and Sharp, 1999). 5-HT modulates mood, anger, aggression, stress, sleep, body temperature, and metabolism (Nelson and Trainor, 2007; Grahn et al., 1999; Maier and Watkins, 2005; Sørensen et al., 2000). ${ }^{6}$

\subsection{Triggering of unlearned behaviors}

In probably all animals, evolution has led to the emergence of a number of stereotyped unlearned basic behaviours that are triggered when specific stimuli are perceived. For example, these behaviours lead a hungry rat to approach food as soon as this is perceived (e.g., smelled), and, once it is close to the mouth, to ingest it. Similarly, a rat will regularly perform a rearing behaviour directed to detect predators. In case the rat spots one, it will freeze if the predator is far or startle and then engage in flight or fight behaviors if the predator is close.

Amg plays two important functions in the selection of these behaviors. First, it allows the anticipatory execution of these behaviours in correspondence to previously neutral stimuli which predict the appearence of the stimuli that innately trigger the behaviours. For example, the sight of a landmark previously associated with food might trigger an approaching behaviour directed to it and this might allow obtaining the food; a particular smell associated with a predator might trigger a startle reflex and then a flight behaviour. Second, Amg modulates the triggering of these basic behaviours on the basis of relevant internal states. For example, a rat can stop executing a feeding behaviour if it becomes satiated, or can decide whether to fight or flight on the basis of its perceived internal state.

The Amg exerts a control on unlearned behaviours on the basis of a complex network of connections that CEA has with various nuclei (figure 8). So, for example, CEA can trigger freezing and fleeing behaviours via PAG (Bandler et al., 2000; Davis and Whalen, 2001), and the startle reflex via NRPC (Davis

\footnotetext{
${ }^{4}$ NE plays an important function also within the sympathetic system and is also released as a hormone in the blood by adrenal medulla.

${ }^{5}$ Ach is also used in the peripheral nervous system to activate muscles.

${ }^{6} 5$-HT is also a peripheral signal mediator, in particular within the guts autonomic system.
} 
and Whalen, 2001). Furthermore, CEA might also exploit more indirect mechanisms based on DA to modulate the triggering and execution of feeding, rearing and approaching behaviours. In particular, CEA might enhance feeding behaviours via the dopaminergic modulation of NAccS-VP-LH pathway through VTA (Ahn and Phillips, 2002; Wyvell and Berridge, 2000; Smith and Berridge, 2005; Tindell et al., 2006). Similarly, rearing seems to be performed on the basis of a striato-cortical loop passing through DLS-PMC-MC and might be modulated by CEA via a DA influence passing through SNpc (Han et al., 1997). In a similar way, the fundamental behaviour of approaching, which plays a central role in the adaptation of organisms as it allows them to get in contact with the needed resources scattered in the environment, is performed via a second striato-cortical loop involving NAccC and $\mathrm{AC}$, which can be influenced by CEA through DA produced via connections to VTA (Parkinson et al., 2000; Cardinal et al., 2003). Note how these mechanisms differ from the direct triggering of basic behaviors, like the one that passes through PAG: in fact, they imply an existing tendency to perform the behaviour (for example, moving towards a perceived object), and a modulation by Amg of this tendency (this modulation is thought to be performed through the VTA-NAcc or the nigro-striatal dopaminergic connections). Finally, note that the difference between the direct triggering of basic behaviors (e.g., freezing) and the modulation of the probability of performing some generic behavior (e.g., approaching) might constitute a general difference between negative-conditioning and appetitive-conditioning phenomena.

\section{The roles of amygdala in cognitive processes}

Thanks to its capacity to trigger basic affective responses on the basis of conditioning processes, amygdala also evolved the capacity to act as a link between affective processes and cognitive ones, thus allowing the development of important new functionalities. In this section we discuss three fundamental new cognitive functions allowed (or enhanced) by Amg: affective labeling (5.1), goal-directed behavior (5.2), and planning and decision making (5.3).

\subsection{Affective labeling}

One of the most important memory functions of the brain is its capacity to quickly store specific events characterised by unique and arbitrary configurations of objects and events in space. This capability plays a very important role for organisms' survival as it allows them to store important information on the basis of a few experiences or even a single experience.

This functionality relies heavily on Hip and its peculiar anatomical and physiological properties. These properties have been specified at a theoretical level in McClelland et al. (1995), have been modelled in Alvarez and Squire (1994), and can be summarised as follows (cf. Rolls and Kesner, 2006): (a) Hip has important reciprocal connections with many associative cortical areas (e.g., 


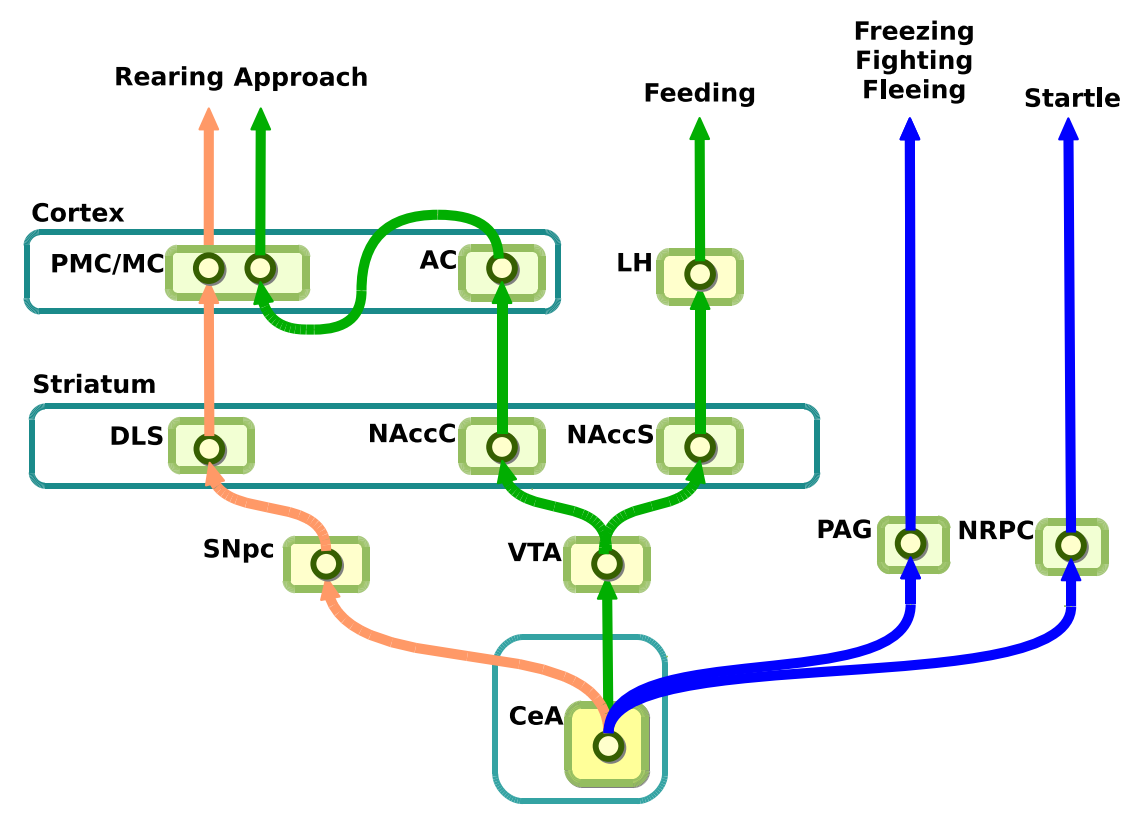

Fig. 8. Triggering of unlearned behaviors: schematization of how amygdala contributes to the triggering of unlearned behaviors via different sub-cortical and cortical brain areas. Acronyms: AC (anterior cingulate cortex), CeA (central amygdaloid nucleus), DLS (dorsolateral striatum), LH (lateral hypothalamus), MC (motor cortex), NAccC (nucleus accumbens core), NAccS (nucleus accumbens shell), NRPC (nucleus reticularis pontis caudalis), PAG (periaqueductal gray), PMC (premotor cortex), SNpc (substantia nigra, pars compacta), VTA (ventral tegmental area).

PFC, IT, PPC) and sub-cortical nuclei (e.g., NAcc and Amg); (b) Hip neurons have massive lateral connectivity; (c) Hip is one of the brain loci where rapid associative learning leading to Long Term Potentiation is strongly present; (d) Hip has been shown to reactivate during sleeping (McClelland et al., 1995; Eschenko et al., 2008).

On this basis, McClelland et al. (1995) suggested that Hip plays an important role in episodic memory acquisition and consolidation. In particular, Hip can rapidly form neural associations between sub-clusters of its neurons and several different multimodal activation patterns that take place in different brain areas at the same time. Consequently, Hip can form representations of any arbitrary polimodal pattern existing at a certain time. According to the authors, the later spontaneous reactivation of Hip clusters (e.g., during sleep) causes the reactivation of the corresponding patterns located in the various areas of the brain and so allows the formation of direct connections between them ('consolidation'). With consolidation, the patterns initially stored in Hip either fade away (within days/months) or continue to contribute, at least in part, to mem- 


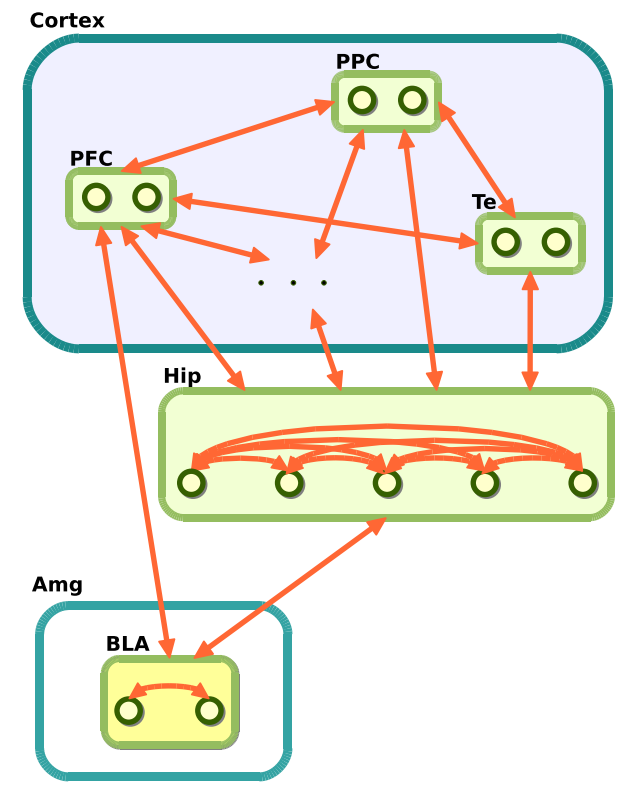

Fig. 9. Affective labeling: schematization of how amygdala 'tags' memories stored in Hip and cortex through affective evaluations of stimuli and episodes. Plastic connections and innate connections are respectively indicated with gray and black arrows. Acronyms: Amg (amygdala), BLA (basolateral amygdaloid complex), Hip (hippocpampus), PFC (prefrontal cortex), PPC (posterior parietal cortex), Te (temporal cortex).

ory recall processes (Rolls and Kesner, 2006). The slow speed and intermixed order with which consolidation of different experiences takes place within the Hip target areas allows the formation of semantic long-term memories having a high degree of generalisation within them. With repetition, this allows such areas to capture the common structure existing in the different experienced episodes.

BLA plays at least two important roles in the formation of episodic memories within Hip. First of all, it is important that only the experiences with high relevance for survival and reproduction are stored by Hip. Being the pivot of affective regulations, Amg contains the information needed to decide which events, either with a positive or negative value, might have a high biological relevance, and therefore deserve to be stored in Hip. This first function is likely played by the Amg on the basis of its influence on neuromodulators (cf. section 4.2), which play a very important role in Hip learning.

A second, more direct, function played by Amg in episodic and semantic memories is based on the massive reciprocal connections it forms with Hip. These connections allow Amg to furnish Hip with the current affective context, which is to be integrated with the other cognitive components that form 
the episodes to be stored. With the consolidation process driven by Hip, the information stored within Amg gets directly associated with other cortical and sub-cortical areas with which it is directly or indirectly (especially via PFC) connected. In this way, such information comes to play the role of a sort of affective tag associated with the stored episodes. This association allows two fundamental processes to take place. First, it allows affective reactions taking place withing Amg to contribute to the recall of memories stored within the Hip or within the areas with which the current affective context has been associated during consolidation (LaBar and Cabeza, 2006; Phelps, 2004). Second, when Hip, or the areas linked between them during consolidation, recall particular episodes, their association with Amg allows them to reactivate the affective valence of such episodes within Amg itself. This in turn can lead to: (a) triggering of the brain and body regulations that are suitable for the given episode (this might be important if the current situation is similar to the recalled episode); (b) informing the Hip (via reciprocal connections from Amg) on the biological saliency of the recalled episode (this might be important when Hip itself exerts a direct or indirect influence on action).

So, for example, imagine a rat has experienced an attack from a predator after having perceived a particular noise in a certain location of the environment. A later sight of the same place might trigger the recall of the noise memory (and hence trigger a useful priming effect which would facilitate the detection of the noise) and this might activate the related memories of the negative effects of the attack within Amg (thanks to a CS-US association). In turn, this reactivation might not only trigger a suitable regulation of the body (e.g., making the body ready for fleeing or fighting) and the brain (e.g., enhancing attentive processes and general arousal), but also contribute to the recall of further memories within Hip (or within the areas connected by it during consolidation), for example the path followed to reach a safe place after the attack.

\subsection{Goal-directed behaviors}

As mentioned in section 3, instrumental (or 'operant') learning represents, aside Pavlovian learning, one of the two fundamental processes underling individual learning in complex organisms (Thorndike, 1911; Skinner, 1938; Domjan, 2006). As we have seen, instrumental learning allows organisms to form stable S-R associations between stimuli and responses, initially produced by chance, if the latter allow obtaining rewards or avoiding punishments. The acquisition of S$\mathrm{R}$ associations is well captured by reinforcement learning models (Barto, 1995; Sutton and Barto, 1998). Such S-R associations are acquired only with prolonged training and form efficient but rather rigid 'habits' that are performed independently of the current value of the pursued outcome (e.g., food, see below).

Basal ganglia are considered to be the main locus where operant conditioning associations take place. In particular, the macro-loop formed by DLS with cortex (in particular PMC/MC) via the Th, is known to play a fundamental role in both the acquisition and the expression of S-R associations (Yin and Knowlton, 2006). 


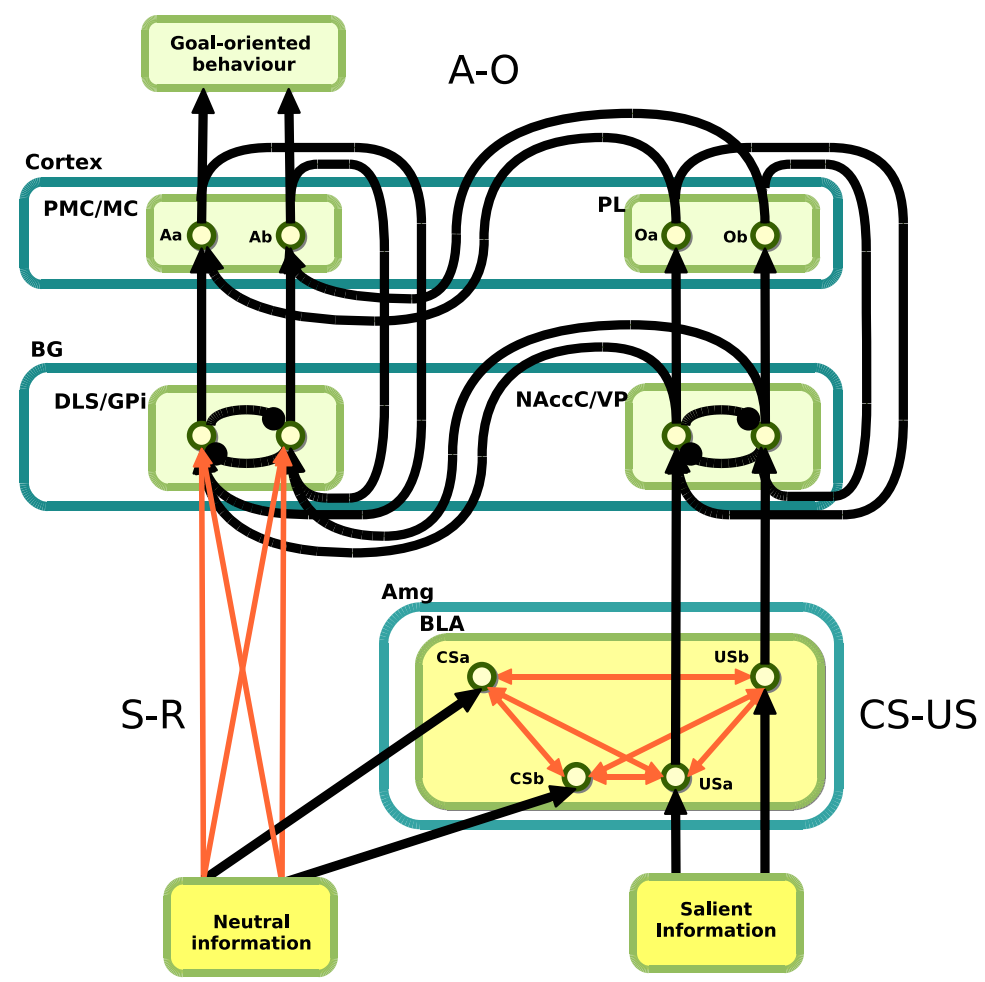

Fig. 10. Goal-directed behaviour: schematization of how amygdala contributes to bias the selection of instrumentally-acquired stimuli. Plastic connections and innate connections are respectively indicated with thin and thick arrows. Circle edges denote inhibitory connections whilst arrow edges denote excitatory connections. Acronyms: Amg (amygdala), BG (basal ganglia), BLA (basolateral amygdaloid complex), DLS (dorsolateral striatum), GPi (globus pallidus, internal segment), MC (motor cortex), NAccC (nucleus accumbens core), PL (prelimbic cortex), PMC (premotor cortex), VP (ventral pallidum).

Other portions of BG, in particular the two macro striato-thalamo-cortical loops DMS-PFC/PPC and NAcc/vmPFC, play a rather different role. In particular, the DMS-PFC/PPC loop plays an important role in the initial phases of learning, when the S-R habits are not formed yet (Yin and Knowlton, 2006). The NAcc/vmPFC loop, which is particularly relevant here for the strong projections it gets from the BLA, is very important for the selection of an action on the basis of the current value of its outcome (action-outcome, or A-O, associations), for example the current potential value of the pursued food (Balleine and Dickinson, 1998).

The behaviors modulated on the basis of A-O associations have a typical goal-directed nature in that they lead to select an action on the basis of a rela- 
tion which goes from the outcomes of the action to the action itself and so inverts the temporal and causal relationship existing between them (actions cause the achievement of outcomes; cf. Hommel, 2005; Pezzulo et al., 2007). In this respect, the goal-directed modulation of the selection of instrumental behaviours considered here represents the first fundamental departure from the S-R habit scheme. This departure reaches its maximum degree of development with planning and complex decision making, which will be described in section 5.3.

The functionality accomplished by the A-O mechanisms has a fundamental adaptive role: it allows internal body states and needs, via the MEA-BLA pathway, to bias the selection of different instrumentally-acquired behaviors which might be triggered in a given situation. An example of this is elegantly captured by instrumental devaluation experiments in which a rat that can perform two instrumentally acquired actions to achieve two different outcomes (e.g., different resources satisfying different needs). In this case, rats are able to appropriately select which action to perform on the basis of the current configuration of their internal states and needs. These mechanisms clearly add a great flexibility to a rigid stimulus-response system that is insensible to the current state of the animal (ses section 4.1 above).

Balleine and Dickinson (1998) pioneered a whole new research agenda devoted to the study of A-O behaviours and to contrast them with S-R behaviours traditionally studied within the behaviourist approach. These authors give an operational definition of goal-directed behaviours based on two classes of experiments:

1. Goal-directed behaviours are sensitive to the degradation of the $A-O$ contingency, that is the strength of the causal relationship existing between the performance of an action and the achievement of the related outcome (the contingency strength is measured on the basis of the relation existing between the probabilities of obtaining the outcome with and without the action). If the triggering of an instrumentally acquired action is sensitive to the degradation of the A-O contingency (e.g., when the outcome is delivered non-contingently to the action), then the action is considered to be goal-directed; otherwise it is considered to have become a habit (Balleine and Dickinson, 1998).

2. Goal-directed behaviours are immediately (i.e. without the need of a new training) sensitive to manipulations of the value that the organism assigns to the outcome (Balleine and Dickinson, 1998). For example, in a typical instrumental devaluation experiment (Balleine et al., 2003) one of two foods ('Food1' and 'Food2') previously used to form two instrumental associations, 'PressLever1-Food1' and 'PressLever2-Food2' , is 'devaluated' by allowing the rat to freely access it (e.g., Food1). In a successive test the rat is exposed to both Lever1 and Lever2. If the rat has a bias to select the lever that is associated to the non-devaluated food, then the behavior is considered to be goal-directed, otherwise it is considered a habit.

Figure 10 presents a scheme that illustrates the most important mechanisms involved in goal-directed behaviour, for example the instrumental devalua- 
tion experiment illustrated above (a simple version of the model based on this scheme that reproduce some of the available data on real rats has already been published in Mannella et al., 2007, 2009; the model is now being further refined so to reproduce a higher number of data, in particular on lesions). In the model, stimulus-response (S-R) associations acquired on the basis of external rewards are stored in the left part of the system shown in the figure: in particular, in the connections that bring the information on neutral stimuli to the DLS/GPi pathway of the basal ganglia. This sub-system allows the rat to acquire the two instrumental action 'PressLever1-Food1' and 'PressLever2-Food2' in the first phase of the experiment, when the two levers are presented separately. In the test phase, which takes place after one of the two foods has been devaluated with free access to it (say Food1), the two levers are presented together. In this condition the rat exhibits a strong tendency to select the lever that is associated to the non-devaluated food (Lever2) thanks to the biasing effects that Amg indirectly exerts on the S-R system through the NAccC/VP-PL pathway. This effect depends on three fundamental mechanisms:

1. While in the first phase of the experiment the rat instrumentally acquires the S-R responses, the creation of the contingency between the observation of each lever and the following reception of the corresponding food allows Amg to form two CS-US associations: Lever1-Food1 and Lever2-Food2.

2. In the devaluation phase, when the rat gets satiated with one food (e.g., Food1), the resulting internal state inhibits the corresponding representation of food (US) within BLA.

3. When in the last phase the rat is exposed to the two levers, only one of the two representations of the levers (CSs) within Amg succeed to activate the corresponding US representation and so to exert an influence on the corresponding S-R association via NAccC (Corbit et al., 2001). Importantly, the actual biasing effects of Amg on instrumental behaviors, which instantiate the A-O associations within the model, can be performed both via the striato-nigro-striatal connections ('dopamine spirals', Haber, 2003) and via PFC (in particular, PL: Corbit and Balleine, 2003).

These mechanisms capture the essence of how Amg can increase the adaptation of animals thanks to its capacity of forming CS-US associations (within BLA) and of modulating their activation on the basis of internal states (through MEA). In particular, this section has shown how these capabilities add an important flexibility to the selection of inherently-rigid habits acquired with instrument conditioning.

\subsection{Planning and decision making}

Planning and decision making can be considered the hallmark of complex cognition in mammals. Planning consists in the internal generation of trajectories in the space of possible future environment states on the basis of available actions (Dagher et al., 2001). Decision making involves the selection among alternative 

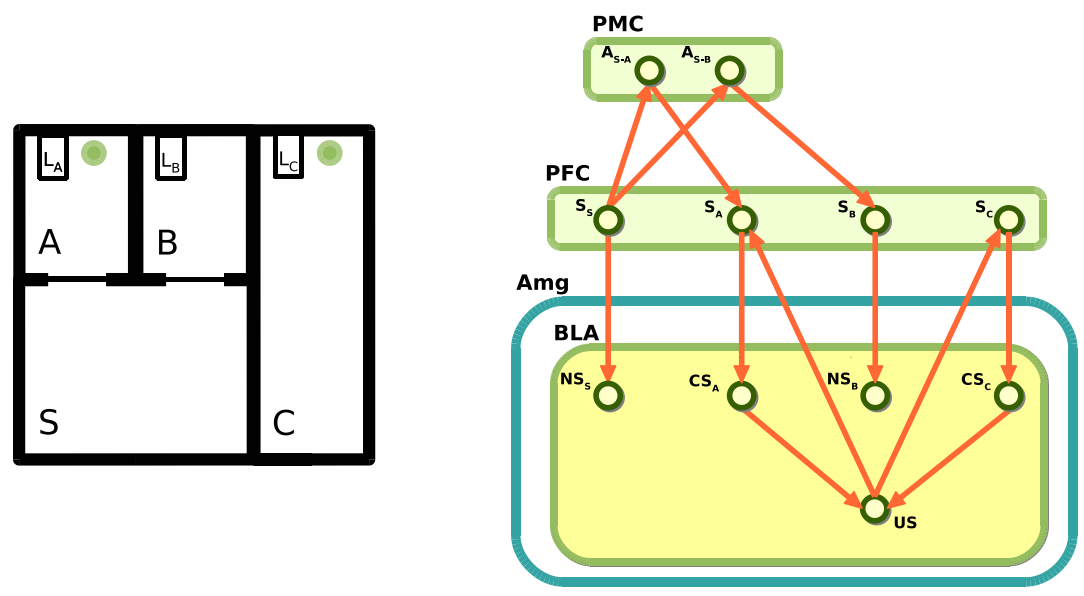

Fig. 11. Planning: schematization of how amygdala contributes to planning by furnishing values to anticipated states. Left: a hypothetical task involving planning. Right: a possible model to tackle the task. All connections in the model are learned in the various phases of the process (see text). $A, B, C, S$ states corresponding to being in different chambers; $L$ : lever; $N S$ : neutral stimulus; $C S$ : conditioned stimulus; $S$ : state; $A$ : action leading from one state to another. Acronyms: Amg (amygdala), BLA (basolateral amygdaloid complex), PFC (prefrontal cortex), PMC (premotor cortex).

actions on the basis of a calculation of the actions' possible consequences, their values, and their estimated probabilities of actually happening (Bechara et al., 1996). Thus, the core functionality underlying both planning and decision making seems to be the capacity of producing internal representations of possible future states which might be experienced as a consequence of one's own actions (Miller and Cohen, 2001).

The development of these capabilities has reached the maximum level of sophistication in humans thanks to the evolution of an exceptionally extended and complex PFC cortex (and, probably, thanks to the use of language as a cognitive tool: see Mirolli and Parisi, 2009, 2010). In this respect, the PFC represents the brain area governing behaviour at the maximum level of abstraction and involving the longest time scope of cognition (Miller and Cohen, 2001).

The amygdala'a role in planning and decision making is based on its capacity to provide the values of the imagined possible states in order to bias the selection of a course of actions towards the maximization of the probability of achieving biological advantages while reducing physical damages and other costs to a minimum (Kringelbach and Rolls, 2004). In this respect, imagine a rat which has previously experienced food in a certain place in the environment but, on the way to it, it smells the presence of a predator, for example a cat. The rat has to decide whether to keep on moving forward towards the food, or, say, to detour and reach the food by following a much longer route. Crucially, this decision should depend on several factors, such as the chances of encountering 
the predator along the direct path (which might be signalled by the intensity of the predator's smell), the anticipated energy spent in the detour, the knowledge of the path to be followed in the case of the detour, the information from the body related to the current level of hunger, and so on.

A possible experiment capturing this type of situation, which is inspired by response-preconditioning experiments (St Claire-Smith and MacLaren, 1983), is as follows. Consider a rat that is set in a chamber $\mathrm{S}$ from which it can access two chambers $A$ and $B$ by entering their respective gated entrances (the gates prevent the rat from seeing the inside of $A$ and $B$ from $S$ ). Now assume that each of the two chambers A and B contains a lever, respectively LeverA and LeverB, and that the rat is left free to explore this environment for a prolonged period of time (this allows the rat to experience the passage between $\mathrm{S}, \mathrm{A}$ and $\mathrm{B}$, and to experience the levers with not reward). Also assume that the rat can experience a further level, LeverC, in a chamber $C$ which does not communicate with either S, A or B. Finally, assume that in a second training phase, composed of three sub-phases, the same rat experiences: (a) a LeverA-food association in A; (b) a LeverC-food association in C; (c) a LeverB-no food condition in B. Now, if in a third phase the rat is set in $S$, one might expect that the rat would exhibit the tendency to enter A more than B, as in A it would expect to see LeverA, which has been associated with food in the second phase.

Figure 11 shows a sketch of a model which might be implemented to reproduce the role of Amg and PFC (in particular OFC, IL, and PL) in the described experiment. The figure shows that the experience of the first phase might allow the rat to form associations between the representations of both chambers $\mathrm{A}$ and $\mathrm{B}$ and the representation of chamber $\mathrm{S}$ in PFC, linked, respectively, by the representations of the actions that lead from $\mathrm{S}$ to $\mathrm{A}\left(A_{S-A}\right)$ and from $\mathrm{S}$ to $\mathrm{B}$ $\left(A_{S-B}\right)$. Furthermore, suppose that appropriate associations have been formed also between the representations of $\mathrm{S}, \mathrm{A}, \mathrm{B}$, and $\mathrm{C}$ in PFC and the corresponding representations in $\mathrm{Amg}\left(C S_{S}, C S_{A}, C S_{B}\right.$, and $\left.C S_{C}\right)$. When, in the third phase, the rat is set in $S$, the PFC representation of $S$ might cause an anticipatory reactivation of the internal representations of $\mathrm{A}$ and $\mathrm{B}$, via $A_{S-A}$ and $A_{S-B}$, respectively, but not of $C$, as the rat has not experienced any action which can lead from $S$ to C. The activation of $S_{A}$ and $S_{B}$ in PFC would cause the corresponding representations within $\mathrm{Amg}$ to be activated $\left(C S_{A}\right.$ and $\left.C S_{B}\right)$. Since only $C S_{A}$ is able to reactivate the representation of food (US), this might produce a feedback signal to the PFC representation of $\mathrm{A}$, which would strengthen the activation of $A_{S-A}$ in comparison to $A_{S-B}$. This might lead to the selection and performance of action $A_{S-A}$ versus $A_{S-B}$ at the level of the striato-cortical loops (in particular, DMS and DLS, and PMC and MC), with which PFC is both directly and indirectly connected.

Amg and PFC, in particular BLA and OFC, play also a key role in complex decision making. This is demonstrated, for example, by the experiments of Winstanley et al. (2004), who trained rats with two levers, one producing a small immediate amount of food and the second one producing a larger but delayed amount of food (see also Mobini et al., 2002). Interestingly, rats which 
received a post-training lesion of BLA exhibited a higher tendency to select the immediate-food lever in comparison to shams whereas rats which received a post-training lesion of OFC exhibited a higher tendency to select the delayedfood lever. Although a commonly accepted explanation of these experiments is not available yet (cf. Mobini et al., 2002; Winstanley et al., 2004; Schoenbaum et al., 2007), they show that OFC and BLA play a central role in complex decision making.

It is interesting to relate these data on rats with those on complex decision making in humans. For example, humans with a damaged Amg/OFC/vmPFC perform poorly in tasks requiring the integration of information about imagined gains and costs in the financial domain. Bechara et al. (1994) developed a task, the Iowa Gambling Task (IGT), specifically for studying this kind of dysfunctions. In this task, the subjects have to choose a card from one of two decks: one deck produces low monetary gains with a high probability, while the other produces high gains with a low probability but also high losses. On average, the net gain with the first deck is higher than that with the second deck. Whereas control subjects learn to choose cards from the first deck and also exhibit an increased skin conductance before selecting a card from the second deck, patients with damage to either the Amg or the vmPFC tend to prefer the high-risk deck and also fail to show increased skin conductance.

Bechara et al. (1996) have proposed that Amg and vmPFC play a central role in guiding choices in the IGT. The idea, which is in line with our proposal discussed in relation to figure 11, is that PFC anticipates possible future events (e.g., financial gains or damages) which are evaluated by the PFC-Amg re-entrant loops thanks to the capacity of Amg of activating the body reactions that would follow from the actual experience of such events. In this respect, these affective body reactions play the role of 'somatic markers' of possible events that, once propagated back to PFC, support the selection or rejection of the alternative courses of available action.

\section{Conclusion}

The amygdala is a brain system that plays a fundamental role in the affective regulation of body, brain, and behaviour. This paper has presented the general principles that might underlie the inner functioning of the amygdala, and has illustrated how these principles might allow the amygdala to play a key role in several affective and cognitive processes. In particular, we have shown how the amygdala is capable of integrating information from internal states, innately relevant stimuli, and innately neutral stimuli on the basis of three core mechanisms. First, the amygdala directly associates important basic reactions (e.g., approaching and salivation) that are innately triggered by biologically relevant stimuli (e.g., food) to neutral stimuli (e.g., the sight of a landmark signaling the presence of food). Second, the amygdala associates representations of neutral stimuli (e.g., of the landmark) to representations of biologically relevant stimuli (e.g., the food), thus transferring all the properties of the latter to the former. 
Third, the amygdala modulates these associations on the basis of the internal states of the animal (e.g., satiation can inhibit both the triggering of salivation caused by the sight of a landmark predicting food and the re-activation of the internal representation of the food itself).

Furthermore, we have shown how these basic functioning principles of the amygdala allow it to play a role in the regulation of three fundamental classes of affetive processes, namely: (a) the regulation of body states; (b) the regulation of brain states via the principal neuromodulators; (c) the triggering of a number of basic behaviours relevant for the organism's survival and reproduction.

Finally, we have shown how the same mechanisms allow the amygdala to exert an important affective influence on cognition, thus permitting the emergence of three high-level cognitive processes: (a) the affective labeling of memories with the biological valence of stimuli and episodes; (b) the biasing of the selection of instrumental responses on the basis of the current valence of their expected outcomes; (c) the biasing of planning and decision making processes through the 'marking' of possible states and actions with their affective valence.

Both the overall picture and the specific claims proposed in the article have been developed by trying to fulfill two main constraints: on one hand, the biological constraints coming from the currently available empirical knowledge; on the other hand, the computational constraints that depend on trying to build working systemic models which can reproduce and explain empirical phenomena. Moreover, further sources of constraints came from assuming a functional/adaptive stance, and the will to isolate general principles, rather than ad-hoc explanations of single phenomena/experiments.

In this respect, it is important to note that while some of the ideas presented here can be considered as acquired knowledge in the field of affective neuroscience, other ideas constitute original hypotheses that are well supported by the available empirical knowledge, and still others (hopefully a minority) represent more speculative ideas that will turn out to be wrong from an empirical point of view. We think that the speculative nature of some of the ideas presented here does not represent a serious problem since the value of the paper is to be found not in any of the specific hypotheses we have presented but rather in the whole picture that we have provided regarding the biological organization of some important adaptive behavior. This picture has been centered on the amygdala because this plays a fundamental role in interfacing motivational/emotional processes and cognitive processes.

We hope that the detailed and coherent picture provided here will contribute on one hand to foster more theoretically oriented research within affective neuroscience and on the other hand to produce more biologically-informed computational models of the affective aspects of adaptive behavior. More in general, we hope that this kind of work will foster an increase in the interactions between the empirical and the synthetic sciences devoted to study (the affective aspects of) the brain and behavior. 


\section{Appendix: Acronyms}

This appendix presents the acronyms used throughout the paper.

\begin{tabular}{|c|c|c|c|}
\hline \multicolumn{2}{|c|}{ Neuromodulators: } & \multirow{3}{*}{$\begin{array}{l}\text { Lvl } \\
\text { Lvm }\end{array}$} & \multirow{3}{*}{$\begin{array}{l}\text { Lateral ventrolateral amygdaloid nu- } \\
\text { cleus } \\
\text { Lateral ventromedial amygdaloid nu- } \\
\text { cleus }\end{array}$} \\
\hline $\mathrm{ACh}$ & Acetylcholine & & \\
\hline DA & Dopamine & & \\
\hline NE & Norepinephrine & MB & Midbrain \\
\hline \multirow[t]{2}{*}{$5 \mathrm{HT}$} & Serotonine & $\mathrm{MC}$ & Motor cortex \\
\hline & & MEA & Medial extended amygdala \\
\hline \multirow{2}{*}{\multicolumn{2}{|c|}{ Anatomic components: }} & MEV & Midbrain trigeminal nucleus \\
\hline & & MG & Medial geniculate nucleus \\
\hline $\mathrm{AB}$ & Accessory basal amygdaloid nucleus & Md & Medial amygdaloid nucleus, dorsal \\
\hline AC & Anterior cingulate cortex & & part \\
\hline AMB & Nucleus ambiguus & $\mathrm{MeA}$ & Medial amygdaloid nucleus \\
\hline Amg & Amygdala & Mv & Medial amygdaloid nucleus, ventral \\
\hline $\mathrm{B}^{\circ}$ & Basal amygdaloid nucleus & & part \\
\hline BG & Basal ganglia & NAcc & Nucleus accumbens \\
\hline BL & Basolateral amygdaloid nucleus & $\mathrm{NAccC}$ & Nucleus accumbens core \\
\hline BLA & Basolateral amygdaloid complex & NAccS & Nucleus accumbens shell \\
\hline BNST & Bed Nucleus of the stria terminalis & NRPC & Nucleus reticularis pontis caudalis \\
\hline $\mathrm{CB}$ & Cerebellum & NST & Nucleus of the solitary tract \\
\hline CEA & Central extended amygdala & OB & Olfactory bulb \\
\hline $\mathrm{CeA}$ & Central amygdaloid nucleus & OFC & Orbitofrontal cortex \\
\hline CL & Central lateral amygdaloid nucleus & PAG & Periaqueductal gray \\
\hline \multirow[t]{2}{*}{ CLC } & Central amygdaloid nucleus, lateral & PAL & Pallidum \\
\hline & capsular suddivision & PB & Parabrachial nucleus \\
\hline $\mathrm{CM}$ & Central medial amygdaloid nucleus & PC & Piriform cortex \\
\hline DI & Disgranular insular cortex & PFC & Prefrontal cortex \\
\hline \multirow[t]{2}{*}{ DIg } & Disgranular insular cortex, gustatory & Pit & Pituitary gland \\
\hline & part & PL & Prelimbic cortex \\
\hline \multirow[t]{2}{*}{ DIv } & Disgranular insular cortex, visceral & PMC & Premotor cortex \\
\hline & part & $\mathrm{PO}$ & Preoptic nucleus of hypothalamus \\
\hline DLS & Dorsolateral striatum & PPC & Posterior parietal cortex \\
\hline DMS & Dorsomedial striatum & PPT & Pedunculopontine tegmental nucleus \\
\hline \multirow[t]{2}{*}{ DMX } & Dorsal motor nucleus of the vagus & PRC & Perirhinal cortex \\
\hline & nerve & PVN & Paraventricular nucleus of hypothala- \\
\hline DR & Dorsal raphe & & mus \\
\hline En & Endopiriform nucleus & $\mathrm{PaRh}$ & Parietal rhinal cortex \\
\hline GPi & Globus pallidus, internal segment & $\mathrm{S}$ & Subiculum \\
\hline Hip & Hippocpampus & SI & Substantia innominata \\
\hline Нyp & Hypothalamus & $\mathrm{SNpc}$ & Substantia nigra, pars compacta \\
\hline IL & Infralimbic cortex & $\mathrm{Te}$ & Temporal cortex \\
\hline ILN & Infralaminar nucleus & $\mathrm{Te} 2$ & Temporal cortex, Area 2 \\
\hline ITC & Intercalated nuclei & $\mathrm{Te} 3$ & Temporal cortex, Area 3 \\
\hline LA & Lateral amygdaloid nucleus & Th & Thalamus \\
\hline LC & Locus coeruleus & VMH & Ventromedial hypothalamus \\
\hline LDT & Laterodorsal tegmental nucleus & vmPFC & Ventromedial prefrontal cortex \\
\hline LG & Lateral geniculate nucleus & VP & Ventral pallidum \\
\hline LH & Lateral hypothalamus & VPMpc & Ventral posteromedial nucleus, parvi- \\
\hline \multirow[t]{2}{*}{$\mathrm{Ld}$} & Lateral dorsal amygdaloid nucleus & & cellular part \\
\hline & & VTA & Ventral tegmental area \\
\hline
\end{tabular}

\section{Acknowledgements}

This research was supported by the EU funded Projects ICEA - Integrating Cognition, Emotion and Autonomy, contract no. FP6-IST-IP-027819, and IM-CLeVeR Intrinsically Motivated Cumulative Learning Versatile Robots, contract no. FP7-ISTIP-231722. 


\section{Bibliography}

Ahn, S. and A. G. Phillips (2002, Dec). Modulation by central and basolateral amygdalar nuclei of dopaminergic correlates of feeding to satiety in the rat nucleus accumbens and medial prefrontal cortex. J Neurosci 22(24), 1095810965.

Alvarez, P. and L. R. Squire (1994, Jul). Memory consolidation and the medial temporal lobe: a simple network model. Proc Natl Acad Sci U S A 91(15), 7041-7045.

Arbib, M. and J. Fellous (2004). Emotions: From brain to robot. Trends in Cognitive Science 8(12), 554-561.

Arias, C. and M. G. Chotro (2007, Mar). Amniotic fluid can act as an appetitive unconditioned stimulus in preweanling rats. Dev Psychobiol 49(2), 139-149.

Aston Jones, G. and J. D. Cohen (2005). An integrative theory of locus coeruleus-norepinephrine function: adaptive gain and optimal performance. Annu Rev Neurosci 28, 403-450.

Avila-Garcia, O. and L. Canamero (2004). Using hormonal feedback to modulate action selection in a competitive scenario. In S. Schaal, A. Ijspeert, A. Billard, S. Vijayakumar, J. Hallam, and J.-A. Meyer (Eds.), From animals to animats 8: Proceedings of the 8th international conference on the simulation of adaptive behavior, Cambridge, MA, pp. 243-252. MIT Press.

Balkenius, C. (1993). The roots of motivations. In J.-A. Mayer, H. L. Roitblat, and S. W. Wilson (Eds.), From Animals to Animats II: Proceedings of the Second International Conference on Simulation of Adaptive Behavior, Cambridge, MA. MIT Press.

Balkenius, C. and J. Morén (1999, July). Dynamics of a classical conditioning model. Autonomous Robots 7(1), 41-56.

Balleine, B. W. and A. Dickinson (1998). Goal-directed instrumental action: contingency and incentive learning and their cortical substrates. Neuropharmacology 37(4-5), 407-419.

Balleine, B. W. and S. Killcross (2006, May). Parallel incentive processing: an integrated view of amygdala function. Trends Neurosci 29(5), 272-279.

Balleine, B. W., S. A. Killcross, and A. Dickinson (2003, Jan). The effect of lesions of the basolateral amygdala on instrumental conditioning. J Neurosci 23(2), 666-675.

Bandler, R., K. A. Keay, N. Floyd, and J. Price (2000, Sep). Central circuits mediating patterned autonomic activity during active vs. passive emotional coping. Brain Res Bull 53(1), 95-104.

Bar-Gad, I., G. Morris, and H. Bergman (2003, December). Information processing, dimensionality reduction and reinforcement learning in the basal ganglia. Progress in Neurobiology 71(6), 439-473.

Barnes, N. M. and T. Sharp (1999, Aug). A review of central 5-HT receptors and their function. Neuropharmacology 38(8), 1083-1152. 
Barto, A. (1995). Adaptive critics and the basal ganglia. In J. Houk, J.C. Davis and D. Beiser (Eds.), Models of Information Processing in the Basal Ganglia, pp. 215-232. Cambridge, MA: MIT Press.

Baxter, M. G. and E. A. Murray (2002, Jul). The amygdala and reward. Nature Reviews Neuroscience 3(7), 563-573.

Bechara, A., A. R. Damasio, H. Damasio, and S. W. Anderson (1994). Insensitivity to future consequences following damage to human prefrontal cortex. Cognition 50(1-3), 7-15.

Bechara, A., D. Tranel, H. Damasio, and A. R. Damasio (1996). Failure to respond autonomically to anticipated future outcomes following damage to prefrontal cortex. Cereb Cortex 6(2), 215-225.

Berridge, C. W. and B. D. Waterhouse (2003, Apr). The locus coeruleusnoradrenergic system: modulation of behavioral state and state-dependent cognitive processes. Brain Res Brain Res Rev 42(1), 33-84.

Berridge, K. C. (2007, Apr). The debate over dopamine's role in reward: the case for incentive salience. Psychopharmacology 191(3), 391-431.

Blair, H. T., F. Sotres-Bayon, M. A. P. Moita, and J. E. LeDoux (2005). The lateral amygdala processes the value of conditioned and unconditioned aversive stimuli. Neuroscience 133(2), 561-569.

Cador, M., T. W. Robbins, and B. J. Everitt (1989). Involvement of the amygdala in stimulus-reward associations: interaction with the ventral striatum. Neuroscience 30(1), 77-86.

Canamero, L. (1997). Modeling motivations and emotions as a basis for intelligent behavior. In W. Lewis Johnson (Ed.), Proceedings of the First International Conference on Autonomous Agents, New York, pp. 148-155. The ACM Press.

Canamero, L. (2005). Emotion understanding from the perspective of autonomous robots research. Neural Networks 18, 445-455.

Cardinal, R. N., J. A. Parkinson, J. Hall, and B. J. Everitt (2002, May). Emotion and motivation: the role of the amygdala, ventral striatum, and prefrontal cortex. Neurosci Biobehav Rev 26(3), 321-352.

Cardinal, R. N., J. A. Parkinson, H. D. Marbini, A. J. Toner, T. J. Bussey, T. W. Robbins, and B. J. Everitt (2003, Jun). Role of the anterior cingulate cortex in the control over behavior by Pavlovian conditioned stimuli in rats. Behav Neurosci 117(3), 566-587.

Cecconi, F. and D. Parisi (1993). Neural networks with motivational units. In J. Meyer, H. Roitblat, and S. Wilson (Eds.), From animals to animats 2: Proceedings of the 2nd International Conference on Simulation of Adaptive Behavior, Cambridge, MA, pp. 346-355. MIT Press.

Chen, J., M. Nakamura, T. Kawamura, T. Takahashi, and D. Nakahara (2006, Mar). Roles of pedunculopontine tegmental cholinergic receptors in brain stimulation reward in the rat. Psychopharmacology (Berl) 184(3-4), 514-522.

Corbit, L. H. and B. W. Balleine (2003, Nov). The role of prelimbic cortex in instrumental conditioning. Behav Brain Res 146(1-2), 145-157.

Corbit, L. H., J. L. Muir, and B. W. Balleine (2001, May). The role of the nucleus accumbens in instrumental conditioning: Evidence of a functional dissociation between accumbens core and shell. J Neurosci 21(9), 3251-3260. 
Dagher, A., A. M. Owen, H. Boecker, and D. J. Brooks (2001, May). The role of the striatum and hippocampus in planning: a PET activation study in Parkinson's disease. Brain 124(Pt 5), 1020-1032.

Davis, M. and P. J. Whalen (2001, Jan). The amygdala: vigilance and emotion. Mol Psychiatry 6(1), 13-34.

Dayan, P. (2002). Motivated reinforcement learning. In T. Dietterich, S. Becker, and Z. Ghahramani (Eds.), Advances in Neural Information Processing Systems, Volume 14, Cambridge, MA. MIT Press.

Dayan, P. and B. Balleine (2002). Reward, motivation and reinforcement learning. Neuron 36, 285-298.

De Olmos, J. S., C. A. Beltramino, and G. F. Alheid (2004). Amygdala and extended amygdala. In G. Paxinos (Ed.), The Rat Nervous System. (third ed.). Elsevier accademic press.

Domjan, M. (2006). Principles of Learning and Behaviour. Belmont, CA: Thomson Wadsworth.

Eschenko, O., W. Ramadan, M. Mlle, J. Born, and S. J. Sara (2008). Sustained increase in hippocampal sharp-wave ripple activity during slow-wave sleep after learning. Learn Mem 15(4), 222-228.

Floresco, S. B. (2007, Nov). Dopaminergic regulation of limbic-striatal interplay. J Psychiatry Neurosci 32(6), 400-411.

Fudge, J. L. and A. B. Emiliano (2003). The extended amygdala and the dopamine system: another piece of the dopamine puzzle. J Neuropsychiatry Clin Neurosci 15(3), 306-316.

Fudge, J. L. and S. N. Haber (2000). The central nucleus of the amygdala projection to dopamine subpopulations in primates. Neuroscience 97(3), 479-494.

Grahn, R. E., S. Maswood, M. B. McQueen, L. R. Watkins, and S. F. Maier (1999, Mar). Opioid-dependent effects of inescapable shock on escape behavior and conditioned fear responding are mediated by the dorsal raphe nucleus. Behav Brain Res 99(2), 153-167.

Haber, S. N. (2003, Dec). The primate basal ganglia: parallel and integrative networks. J Chem Neuroanat 26(4), 317-330.

Han, J. S., R. W. McMahan, P. Holland, and M. Gallagher (1997, May). The role of an amygdalo-nigrostriatal pathway in associative learning. J Neurosci 17(10), 3913-3919.

Hatfield, T., J. S. Han, M. Conley, M. Gallagher, and P. Holland (1996, Aug). Neurotoxic lesions of basolateral, but not central, amygdala interfere with pavlovian second-order conditioning and reinforcer devaluation effects. $J$ Neurosci 16(16), 5256-5265.

Hiroi, N. and N. M. White (1991, Jul). The lateral nucleus of the amygdala mediates expression of the amphetamine-produced conditioned place preference. J Neurosci 11(7), 2107-2116.

Hommel, B. (2005, January). Perception in action: Multiple roles of sensory information in action control. Cognitive Processing 6, 3-14.

Hull, C. L. (1943). Principles of behavior. Appleton-century-crofts.

Iversen, S., L. Iversen, and C. B. Saper (2000). The autonomic nervous system and the hypothalamus. In E. R. Kandel, J. H. Schwartz, and T. M. Jessell (Eds.), Principles of neural sciences (fourth ed.). McGraw Hill. 
Jolkkonen, E., R. Miettinen, M. Pikkarainen, and A. Pitkänen (2002). Projections from the amygdaloid complex to the magnocellular cholinergic basal forebrain in rat. Neuroscience 111(1), 133-149.

Jolkkonen, E. and A. Pitkänen (1998, May). Intrinsic connections of the rat amygdaloid complex: projections originating in the central nucleus. J Comp Neurol 395(1), 53-72.

King, B. M. (2006, Nov). Amygdaloid lesion-induced obesity: relation to sexual behavior, olfaction, and the ventromedial hypothalamus. Am J Physiol Regul Integr Comp Physiol 291(5), R1201-R1214.

Knapska, E., K. Radwanska, T. Werka, and L. Kaczmarek (2007, Oct). Functional internal complexity of amygdala: focus on gene activity mapping after behavioral training and drugs of abuse. Physiol Rev 87(4), 1113-1173.

Kringelbach, M. L. and E. T. Rolls (2004, Apr). The functional neuroanatomy of the human orbitofrontal cortex: evidence from neuroimaging and neuropsychology. Prog Neurobiol 72(5), 341-372.

LaBar, K. S. and R. Cabeza (2006, Jan). Cognitive neuroscience of emotional memory. Nat Rev Neurosci 7(1), 54-64.

Lanuza, E., K. Nader, and J. E. LeDoux (2004). Unconditioned stimulus pathways to the amygdala: effects of posterior thalamic and cortical lesions on fear conditioning. Neuroscience 125(2), 305-315.

Lee, H. J., F. Groshek, G. D. Petrovich, J. P. Cantalini, M. Gallagher, and P. C. Holland (2005, Apr). Role of amygdalo-nigral circuitry in conditioning of a visual stimulus paired with food. J Neurosci 25(15), 3881-3888.

Lieberman, D. A. (1993). Learning, behaviour and cognition. Brooks/Cole.

Maier, S. F. and L. R. Watkins (2005). Stressor controllability and learned helplessness: the roles of the dorsal raphe nucleus, serotonin, and corticotropinreleasing factor. Neurosci Biobehav Rev 29(4-5), 829-841.

Mannella, F., M. Mirolli, and G. Baldassarre (2007). The role of amygdala in devaluation: a model tested with a simulated robot. In L. Berthouze, C. G. Prince, M. Littman, H. Kozima, and C. Balkenius (Eds.), Proceedings of the Seventh International Conference on Epigenetic Robotics, pp. 77-84. Lund University Cognitive Studies.

Mannella, F., M. Mirolli, and G. Baldassarre (2009). The interplay of pavlovian and instrumental processes in devaluation experiments: a computational embodied neuroscience model tested with a simulated rat. Cambridge: Cambridge University Press. In press.

Mannella, F., S. Zappacosta, M. Mirolli, and G. Baldassarre (2008). A computational model of the amygdala nuclei's role in second order conditioning. In M. Asada, J. Hallam, J.-A. Meyer, and J. Tani (Eds.), From Amimals to Animals 10, Volume 5040 of LNAI, Berlin, pp. 321-330. Springer Verlag.

Maren, S. (2005, Feb). Building and burying fear memories in the brain. Neuroscientist 11(1), 89-99.

McClelland, J. L., B. L. McNaughton, and R. C. O'Reilly (1995, Jul). Why there are complementary learning systems in the hippocampus and neocortex: insights from the successes and failures of connectionist models of learning and memory. Psychol Rev 102(3), 419-457. 
McDonald, A. J. (1998, Jun). Cortical pathways to the mammalian amygdala. Progresses in Neurobiology 55(3), 257-332.

McDonald, R. J. and N. M. White (1993, Feb). A triple dissociation of memory systems: hippocampus, amygdala, and dorsal striatum. Behav Neurosci 107(1), 3-22.

Miller, E. K. and J. D. Cohen (2001). An integrative theory of prefrontal cortex function. Annu Rev Neurosci 24, 167-202.

Mirolli, M. and D. Parisi (2003). Artificial organisms that sleep. In W. Banzhaf, T. Christaller, P. Dittrich, J. T. Kim, and J. Ziegler (Eds.), Advances in Artificial Life. Proceedings of the 7th European Conference on Artificial Life, Berlin, pp. 377386. Springer Verlag.

Mirolli, M. and D. Parisi (2009). Language as a cognitive tool. Minds and Machines 19(4), 517-528.

Mirolli, M. and D. Parisi (2010). Towards a vygotskyan cognitive robotics: The role of language as a cognitive tool. New Ideas in Psychology.

Mobini, S., S. Body, M.-Y. Ho, C. M. Bradshaw, E. Szabadi, J. F. W. Deakin, and I. M. Anderson (2002, Mar). Effects of lesions of the orbitofrontal cortex on sensitivity to delayed and probabilistic reinforcement. Psychopharmacology (Berl) 160(3), 290-298.

Montebelli, A., C. Herrera, and T. Ziemke (2008). On cognition as dynamical coupling: An analysis of behavioral attractor dynamics. Adaptive Behavior 16(2-3), 182-195.

Murphy, R. e. a. (2002). Emotion-based control of cooperating heterogeneous robots. IEEE Transactions on Robotics and Autonomation 18, 744-757.

Nader, K., P. Majidishad, P. Amorapanth, and J. E. LeDoux (2001). Damage to the lateral and central, but not other, amygdaloid nuclei prevents the acquisition of auditory fear conditioning. Learn Mem 8(3), 156-163.

Nelson, R. J. and B. C. Trainor (2007, Jul). Neural mechanisms of aggression. Nat Rev Neurosci 8(7), 536-546.

Niv, Y., N. Daw, D. Joel, and P. Dayan (2006, Oct). Tonic dopamine: opportunity costs and the control of response vigor. Psychopharmacology (Berl) 191(3), 507520.

O'Reilly, R., M. Frank, T. Hazy, and B. Watz (2007). Pvlv: The primary value and learned value pavlovian learning algorithm. Behavioral Neuroscience 121, $31-49$.

Otani, S., H. Daniel, M.-P. Roisin, and F. Crepel (2003, Nov). Dopaminergic modulation of long-term synaptic plasticity in rat prefrontal neurons. Cereb Cortex 13(11), 1251-1256.

Paré, D., G. J. Quirk, and J. E. LeDoux (2004, Jul). New vistas on amygdala networks in conditioned fear. J Neurophysiol 92(1), 1-9.

Parisi, D. (2004). Internal robotics. Connection Science 16(4), 325-338.

Parkinson, J. A., T. W. Robbins, and B. J. Everitt (2000, Jan). Dissociable roles of the central and basolateral amygdala in appetitive emotional learning. Eur J Neurosci 12(1), 405-413.

Parkinson, J. A., P. J. Willoughby, T. W. Robbins, and B. J. Everitt (2000, Feb). Disconnection of the anterior cingulate cortex and nucleus accumbens core 
impairs Pavlovian approach behavior: further evidence for limbic corticalventral striatopallidal systems. Behav Neurosci 114(1), 42-63.

Pavlov, I. P. (1927). Conditioned Reflexes: An Investigation of the Physiological Activity of the Cerebral Cortex. Oxford University Press.

Petrovich, G. D., P. C. Holland, and M. Gallagher (2005, Sep). Amygdalar and prefrontal pathways to the lateral hypothalamus are activated by a learned cue that stimulates eating. J Neurosci 25(36), 8295-8302.

Petrovich, G. D., B. Setlow, P. C. Holland, and M. Gallagher (2002, Oct). Amygdalo-hypothalamic circuit allows learned cues to override satiety and promote eating. J Neurosci 22(19), 8748-8753.

Peyron, C., J. M. Petit, C. Rampon, M. Jouvet, and P. H. Luppi (1998, Jan). Forebrain afferents to the rat dorsal raphe nucleus demonstrated by retrograde and anterograde tracing methods. Neuroscience 82(2), 443-468.

Pezzulo, G., G. Baldassarre, M. V. Butz, C. Cristiano, and J. Hoffmann (2007). From actions to goals and vice-versa: theoretical analysis and models of the ideomotor principle and tote. In M. V. Butz, O. Sigaud, G. Pezzulo, and G. Baldassarre (Eds.), Anticipatory Behavior in Adaptive Learning Systems: From Brains to Individual and Social Behavior, pp. 73-93. Berlin: Springer-Verlag.

Pfeifer, R. (1993). Studying emotions: Fungus eaters. In Proceedings of the first european conference on artificial life, pp. 916-927.

Phelps, E. A. (2004, Apr). Human emotion and memory: interactions of the amygdala and hippocampal complex. Curr Opin Neurobiol 14(2), 198-202.

Phillips, A. G., G. Vacca, and S. Ahn (2008, Aug). A top-down perspective on dopamine, motivation and memory. Pharmacol Biochem Behav 90(2), 236-249.

Pickens, C. L., M. P. Saddoris, M. Gallagher, and P. C. Holland (2005, Feb). Orbitofrontal lesions impair use of cue-outcome associations in a devaluation task. Behav Neurosci 119(1), 317-322.

Pitkänen, A., E. Jolkkonen, and S. Kemppainen (2000). Anatomic heterogeneity of the rat amygdaloid complex. Folia Morphol (Warsz) 59(1), 1-23.

Pitkänen, A., V. Savander, and J. E. LeDoux (1997, Nov). Organization of intraamygdaloid circuitries in the rat: an emerging framework for understanding functions of the amygdala. Trends Neurosci 20(11), 517-523.

Pitkänen, A., L. Stefanacci, C. R. Farb, G. G. Go, J. E. LeDoux, and D. G. Amaral (1995, May). Intrinsic connections of the rat amygdaloid complex: projections originating in the lateral nucleus. J Comp Neurol 356(2), 288-310.

Price, J. L. (2003, Apr). Comparative aspects of amygdala connectivity. Ann N Y Acad Sci 985, 50-58.

Redgrave, P. and K. Gurney (2006, November). The short-latency dopamine signal: a role in discovering novel actions? Nature Reviews Neuroscience 7(12), 967-975.

Redgrave, P., T. Prescott, and K. Gurney (1999). Is the short latency dopamine response too short to signal reward error? Trends in Neurosciences 22, 146-151.

Richter-Levin, G. (2004, Feb). The amygdala, the hippocampus, and emotional modulation of memory. Neuroscientist 10(1), 31-39.

Roesch, M. and G. Schoenbaum (2006). From associations to expectancies: Orbitofrontal cortex as gateway between the limbic system and representational 
memory. In D. Zald and S. Rauch (Eds.), The Orbitofrontal Cortex, pp. 199-236. Oxford: Oxford University Press.

Rolls, E. T. (2000, Apr). Précis of the brain and emotion. Behavioral and Brain Sciences 23(2), 177-91; discussion 192-233.

Rolls, E. T. and R. P. Kesner (2006, May). A computational theory of hippocampal function, and empirical tests of the theory. Prog Neurobiol 79(1), 1-48.

Romanski, L. M., M. C. Clugnet, F. Bordi, and J. E. LeDoux (1993, Jun). Somatosensory and auditory convergence in the lateral nucleus of the amygdala. Behav Neurosci 107(3), 444-450.

Sah, P., E. S. L. Faber, L. D. M. Armentia, and J. Power (2003, Jul). The amygdaloid complex: anatomy and physiology. Physiol Rev 83(3), 803-834.

Schoenbaum, G., M. P. Saddoris, and T. A. Stalnaker (2007, Dec). Reconciling the roles of orbitofrontal cortex in reversal learning and the encoding of outcome expectancies. Ann N Y Acad Sci 1121, 320-335.

Schoenbaum, G., B. Setlow, M. P. Saddoris, and M. Gallagher (2003, Aug). Encoding predicted outcome and acquired value in orbitofrontal cortex during cue sampling depends upon input from basolateral amygdala. Neuron 39(5), 855-867.

Schultz, W. (2002). Getting formal with dopamine and reward. Neuron 36, 241263.

Schultz, W., P. Dayan, and P. Montague (1997). A neural substrate of prediction and reward. Science 275, 1593-1599.

Schultz, W. and A. Dickinson (2000). Neuronal coding of prediction errors. Annual Reviews Neuroscience 23, 473-500.

Semba, K. and H. C. Fibiger (1992, Sep). Afferent connections of the laterodorsal and the pedunculopontine tegmental nuclei in the rat: a retro- and anterograde transport and immunohistochemical study. J Comp Neurol 323(3), 387410.

Shi, C. and M. Davis (1999, Jan). Pain pathways involved in fear conditioning measured with fear-potentiated startle: lesion studies. Journal of Neuroscience 19(1), 420-430.

Simon, H. A. (1996, October). The Sciences of the Artificial. Cambridge, MA: MIT Press. 3rd Edition.

Skinner, B. (1938). The Behavior of Organisms. New York, NY: Appleton Century Crofs.

Smith, K. S. and K. C. Berridge (2005, Sep). The ventral pallidum and hedonic reward: neurochemical maps of sucrose "liking" and food intake. J Neurosci 25(38), 8637-8649.

Sørensen, E., B. Bjorvatn, and R. Ursin (2000, Sep). Sleep-wake effects following the selective 5-ht(1a) receptor antagonist p-mppi in the freely moving rat. Behav Brain Res 114(1-2), 31-38.

St Claire-Smith, R. and D. MacLaren (1983). Response preconditioning effects. Journal of Experimental Psychology 9, 41-48.

Surmeier, J. D., J. Ding, M. Day, Z. Wang, and W. Shen (2007, May). D1 and D2 dopamine-receptor modulation of striatal glutamatergic signaling in striatal medium spiny neurons. Trends Neurosci 30(5), 228-235. 
Sutton, R. and A. Barto (1998). Reinforcement Learning: An Introduction. Cambridge, MA: MIT Press.

Thompson, R. F., R. Swain, R. Clark, and P. Shinkman (2000, Jun). Intracerebellar conditioning-Brogden and Gantt revisited. Behav. Brain Res. 110(1-2), 3-11.

Thorndike, E. L. (1911). Animal Intelligence. Rutgers, NJ: Transaction Publishers. Tindell, A. J., K. S. Smith, S. Peciña, K. C. Berridge, and W. J. Aldridge (2006, Nov). Ventral pallidum firing codes hedonic reward: when a bad taste turns good. J Neurophysiol 96(5), 2399-2409.

Venditti, A., M. Mirolli, D. Parisi, and G. Baldassarre (2009). A neural-network model of the dynamics of hunger, learning, and action vigor in mice. In R. Serra (Ed.), Proceeding of the Italian Workshop on Artificial Life and Evolutionary Computatoin (WIVACE08). World Scientific. In press.

Winstanley, C. A., D. E. H. Theobald, R. N. Cardinal, and T. W. Robbins (2004, May). Contrasting roles of basolateral amygdala and orbitofrontal cortex in impulsive choice. J Neurosci 24(20), 4718-4722.

Wyvell, C. L. and K. C. Berridge (2000, Nov). Intra-accumbens amphetamine increases the conditioned incentive salience of sucrose reward: enhancement of reward "wanting" without enhanced "liking" or response reinforcement. J Neurosci 20(21), 8122-8130.

Yin, H. H. and B. J. Knowlton (2006). The role of the basal ganglia in habit formation. Nature Reviews Neuroscience 7, 464-476. 